\title{
Meta-analytic evidence for downregulation of the amygdala during
}

working memory maintenance

\author{
Lycia D. de Voogd ${ }^{1}$ and Erno J. Hermans ${ }^{1}$
}

${ }^{1}$ Donders Institute for Brain, Cognition, and Behavior, Radboud University and Radboud University Medical Center, 6500 HB, Nijmegen, The Netherlands

Words in abstract: 229

Words in introduction: 709

Words in discussion: 2172

Figures: 3

Tables: 4

Keywords: meta-analysis, amygdala, working memory, functional MRI, emotion regulation 


\begin{abstract}
The amygdala is a region critically implicated in affective processes. Downregulation of the amygdala is therefore one of the hallmarks of successful emotion regulation. Downregulation is thought to be established through top-down control of the executive control network over the amygdala. Such a reciprocal relationship, however, is not exclusive to cognitive regulation of emotion. It has recently been noted that any cognitively demanding task may downregulate the amygdala, including a standard working memory task. Here, using a coordinate-based meta-analysis based on an activation likelihood estimation (ALE), we examined whether a standard working memory task (i.e., a 2-back task) downregulates the amygdala similarly to a cognitive reappraisal task. Following the PRISMA guidelines, we included a total of 66 studies using a 2-back working memory task and 65 studies using a cognitive reappraisal task. We found that a standard 2-back working memory task indeed systematically downregulates the amygdala, and that deactivated clusters strongly overlap with those observed during a cognitive reappraisal task. This finding has important consequences for the interpretation of the underlying mechanism of the effects of cognitive reappraisal on amygdala activity: downregulation of amygdala during cognitive reappraisal might be due to the cognitively demanding nature of the task and not per se by the act of the reappraisal itself. Moreover, it raises the possibility of applying working memory tasks in a clinical setting as an alternative emotion regulation strategy.
\end{abstract}




\section{Introduction}

Downregulation of the amygdala, a region critically implicated in threat detection (LeDoux, 1996; Öhman, 2005), is one of the hallmarks of successful emotion regulation. Cognitive regulation of emotion is accompanied by activation in the dorsolateral prefrontal cortex (dIPFC), a region that is part of the executive control network (Seeley et al., 2007), and downregulation of the amygdala (Buhle et al., 2014). Since there are little or no direct connections between the dIPFC and the amygdala (Amaral et al., 1992), it is commonly thought that downregulation may occur indirectly, via the ventromedial prefrontal cortex (Buhle et al., 2014).

However, this opposing interplay between the executive control network and the amygdala is not specific for emotion regulation. It has recently been noted that any cognitively demanding task that activates the executive control network may potentially downregulate the amygdala (de Voogd et al., 2018a). Indeed, a downregulation of the amygdala has been observed during the execution of a standard working memory task (de Voogd et al., 2018a, 2018b), with more cognitive load leading to a stronger downregulation (de Voogd, Hermans, et al., 2018). This suggests cognitive demand may play a role in the downregulation of the amygdala that is observed during emotion regulation.

Cognitively demanding tasks have been shown to be accompanied by a downregulation of defensive responses to threat. When participants perform a standard nback working memory paradigm while simultaneously undergoing a threat conditioning paradigm, conditioned responses have been shown to be reduced (Carter et al., 2003). Moreover, threat-potentiated startle responses are decreased when participants perform a working memory paradigm (King and Schaefer, 2011; Vytal et al., 2012). Reductions in these threat-potentiated startle responses are stronger when the cognitive demand is increased (Vytal et al., 2012). Finally, also subjective reports of state anxiety were shown to decrease with increasing cognitive load of a working memory task (Balderston et al., 2016; Vytal et al., 2012).

Lesion studies in humans have indicated that such defensive responses to threat are (partly) dependent on the amygdala (Bechara et al., 1995; Klumpers et al., 2015; LaBar et al., 1995). Therefore, a cognitively demanding task may offer a non-invasive way to impact defensive responses to threat via downregulation of the amygdala. Indeed, threat-induced amygdala responses were shown to be attenuated during the execution of a cognitively 
demanding task (McRae et al., 2010; Price et al., 2013). Even though the general interpretation of such findings is that an initial amygdala activation, in response to the threat, can be downregulated by a cognitively demanding task, other findings show amygdala downregulation can also be observed without the presence of a threat-induced amygdala response (de Voogd et al., 2018a, 2018b). Thus, performing a working memory task alone is sufficient to downregulate the amygdala.

If a working memory task establishes a downregulation of the amygdala and defensive response to threat, it raises the question whether the effects of cognitive reappraisal on the amygdala are driven by cognitive demand. It has been proposed that through a reinterpretation of the threatening situation, with the explicit goal to change the affective impact of the threat, threat-related responses and amygdala reactivity is reduced (Buhle et al., 2014). Alternatively, a downregulation of amygdala during cognitive reappraisal might be due to the cognitively demanding nature of the task and not per se by the act of the reappraisal itself (de Voogd et al., 2018a). It remains unclear, however, whether downregulation of the amygdala is a consistent finding across studies on working memory. More importantly, it is unknown whether there is a systematic difference in amygdala downregulation between a working memory task and cognitive reappraisal.

The aim of this study is therefore to investigate, using a meta-analytic approach, whether working memory tasks downregulate the amygdala, and whether this downregulation is similar to cognitive reappraisal. As a standard working memory task, we opted for a "2-back" working memory task, as there are many studies available that have previously reported an activation (2-back > control) contrast (Lee and Xue, 2018). To test whether a working memory task downregulates the amygdala similar to a cognitive reappraisal task, we conducted an ALE coordinate-based meta-analysis (Eickhoff et al., 2009). We predicted a reduced BOLD signal during a standard 2-back working memory task that would overlap with the reduction in BOLD signal during cognitive reappraisal. 


\section{Methods}

Study and data selection for the ALE meta-analysis

We performed the ALE meta-analysis according to the Preferred Reporting Items for Systematic Reviews and Meta-Analyses (PRISMA) guidelines (Moher et al., 2016). For the PRISMA flow diagram see Fig 1.

2-back working memory

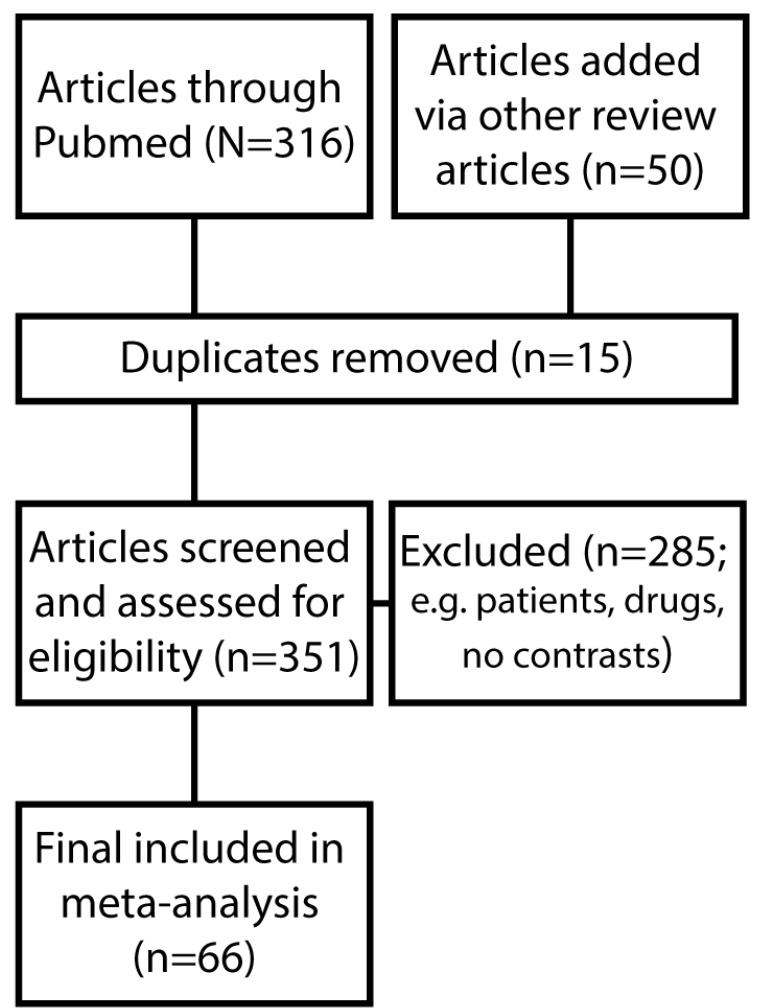

cognitive reappraisal

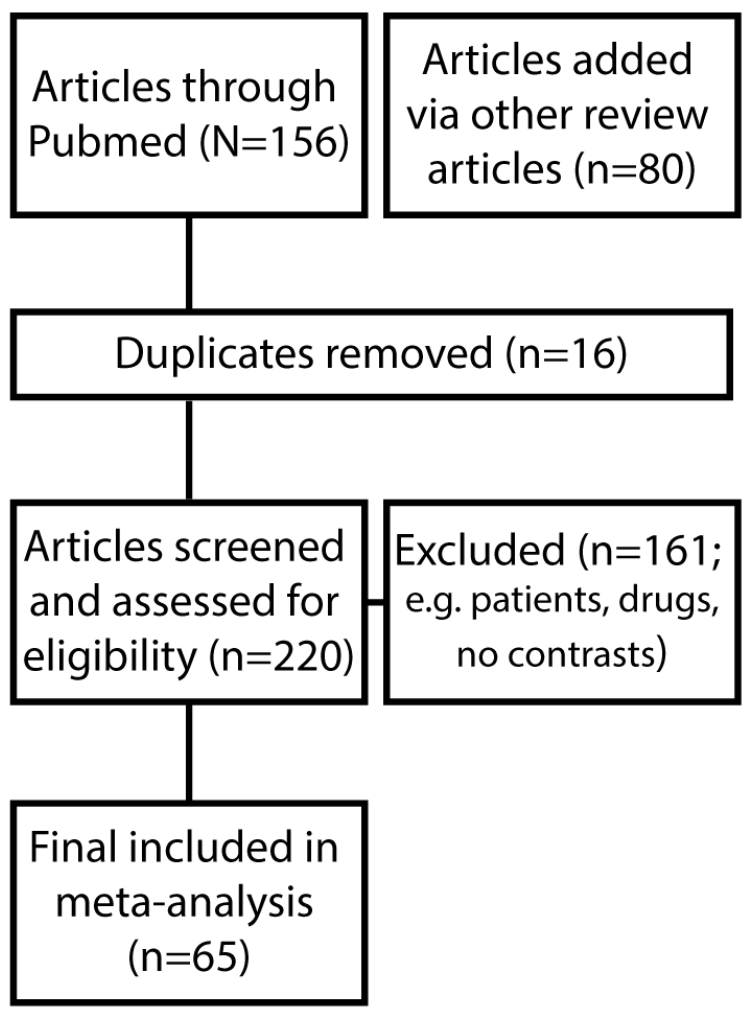

Figure 1 - A flow chart describing the steps used to identify the articles that were included in the ALE meta-analysis.

Eligibility criteria: Peer-reviewed fMRI articles including healthy adult volunteers which included a 2-back working memory or a cognitive reappraisal experiment.

Information sources: The PubMed database (https://www.ncbi.nlm.nih.gov) and other meta-analyses (Buhle et al., 2014; Kohn et al., 2014; Lee and Xue, 2018; Ochsner et al., 2012). 
Search: 1) ((2-back [Title/Abstract]) AND fmri) NOT review [Publication Type], and 2) ((cognitive reappraisal [Title/Abstract]) AND fmri) NOT review [Publication Type]. The search was performed on April 012020.

Study selection: Articles were included based on the following criteria: 1) healthy human adult volunteers (range between 18-45 mean years old). Articles including patient studies with a separate analysis of the control group were included, 2) whole-brain analysis, 3) Region of interest(ROI)-based analysis were excluded, except for the amygdala, 4) reporting of standardized coordinates for activation foci in Montreal Neurological Institute (MNI) or Talairach space, 5) working memory studies including a 2-back condition: the specific modality is reported (see Table 1) OR emotion regulation strategy that involved cognitive reappraisal: the specific technique such as reinterpretation or distancing is reported (See Table 2) 6) general linear model analysis (GLM) involving a 2-back $<>$ control analysis: the control condition such as rest or 0-back is reported (see Table 3 and Table 4) OR GLM analysis involving a Reappraisal < > control analysis: the specific instruction such as view, watch, or attend is reported (See Table 3 and Table 4).

Data collection process: We performed an analysis on 66 working memory studies and 65 emotion regulation studies (See Table 1 and 2). All studies reported an activation contrast (2-back: 954 foci, 80 experiments; cognitive reappraisal: 799 foci, 76 experiments), but 16 (165 foci, 19 experiments) 2-back working memory studies and 29 (289 foci, 34 experiments) emotion regulation studies reported a deactivation contrast. Two 2-back studies included emotional faces as stimuli (See Table 1). Since these can be considered as potentially threatening stimuli, we reran the analysis without these 2 studies to ensure our findings were not driven by these two studies. The results and conclusions remained the same and we therefore included those studies in the final analysis. None of the studies reported a deactivation contrast without an activation contrast.

Data items: We collected the peak coordinates of the selected contrasts for analysis. The focus of this study are the deactivation contrasts (Control > 2-back and Control > Reappraise). We also included the activation contrasts, mainly for comparison purposes to several other meta-analyses as a validation of our procedure. See Table $\mathbf{1}$ and Table $\mathbf{2}$ for the articles included in the ALE meta-analysis. 
Table 1 - An overview of the working memory studies included in the meta-analysis

\begin{tabular}{|c|c|c|c|c|c|c|c|c|c|}
\hline Author & N participans (N Females) & Source & Space & $\mathrm{A} / \mathrm{D}$ & Age $M(S D)$ or range & Domain & Stimuli & Activation contrast & Deactivation contrast \\
\hline Allen et al., 2006 & $10(2 \mathrm{~F})$ & Table 2 & Tal & A & 23-35 range & Visual & Letters & Sham condition (2-back >0-back) & \\
\hline Barch et al., 2007 & $120(70 F)$ & Table 4 & Tal & A & $27.2(10.8)$ & Verbal, Nonverbal & Words, Faces & Working Memory > Encoding & \\
\hline \multirow[t]{2}{*}{ Binder et al., 2006} & $12(5 \mathrm{~F})$ & Table 2 & Tal & A & $23.52(2.52)$ & Verbal & Letters & 2-back >0-back & \\
\hline & $12(5 F)$ & $\begin{array}{l}\text { Table } 3 \\
\text { Table }\end{array}$ & Tal & A & $23.52(2.52)$ & Nonverbal & Abstract texture patterns & 2-back $>0$-back & \\
\hline Bleich-Cohen et al., 2014 & $20(8 \mathrm{~F})$ & $\begin{array}{l}\text { 2a } \\
\text { Table }\end{array}$ & Tal & A & $26.4(2.7)$ & Visual & Achromatic numbers & 2-back $>0$-back & \\
\hline Blokland, et al. 2011 & $319(174 F)$ & s1 & MNI & A & $23.6(1.8)$ & Spatial & Numbers & 2-back $>0$-back & \\
\hline Bustamante et al. 2011 & 15 (OF) & Table 2 & Tal & A & $32.40(7.56)$ & Auditory & Letters & 2-back $>0$-back & \\
\hline Carlson et al. 1998 & $7(3 F)$ & Table 1 & Tal & A & 21.1 & Visuospatial & White squares & 2-back $>0$-back & \\
\hline Chang et al. 2004 & $10(\mathrm{OF})$ & Table 2 & Tal & A & $14.4(3.2)$ & Visuospatial & Letter $\mathrm{O}$ & 2-back $>0$-back & \\
\hline Chang et al., 2010 & 21 (OF) & Table 3 & $\mathrm{MNI}$ & A & $49.7(4.3)$ & Visual & Letters (symbols from the Korean alphabet) & 2-back $>$ rest & \\
\hline Deckersbach et al., 2008 & $17(17 \mathrm{~F})$ & Table 3 & $\mathrm{MNI}$ & A & $25.6(5.9)$ & Visual & Letters & N-back > Fixation (sad/neutral) & \\
\hline Dehghan et al., 2019 & 24(12F) & Tabel 5 & $\mathrm{MNI}$ & $\mathrm{A} / \mathrm{D}$ & $23(2.69)$ & Visual & Letters & 2-back $>0$-back & 0 -back $>2$-back \\
\hline de Voogd et al. 2018 & 24(12F) & Table 1 & $\mathrm{MNI}$ & $A / D$ & $26.95(3.6)$ & Visual & Numbers & 2-back > fixation & fixation $>2$-back \\
\hline Dima et al., 2014 & 40 (20F) & Table II & MNI & A & $31.5(10.4)$ & Visual & Letters & 2-back $>0$-back & \\
\hline Dores et al. 2017 & 10(4F) & Table 2 & Tal & A & $27.10(2.89)$ & Visuospatial & Grid & 2 back $>$ fixation & \\
\hline Drapier et al., 2008 & 20 (10F) & Table 2 & Tal & A & $41.9(11.6)$ & Visual & Letters & 2-back >0-back & \\
\hline Drobyshevsky et al., 2006 & $31(15 F)$ & Table 2 & Tal & A & $41(15.3)$ & Visual & Letters & 2-back $>0$-back & \\
\hline Fernandez-Corcuera et al., 2013 & 41(17F) & Tabel 2 & $\mathrm{MNI}$ & $\mathrm{A} / \mathrm{D}$ & $40.27(9.8)$ & Visual & Letters & 2 back $>$ baseline & baseline $>2$-back \\
\hline Ford et al. 2018 & 32(20F) & Table 3 & $\mathrm{MNI}$ & A & $30-65$ & Visual & Faces/places/tools/body parts & 2-back >0-back & \\
\hline Garrett et al., 2011 & 19 (6F) & Table 3 & Tal & A & $34.85(12.54)$ & Visual & Letters & 2-back $>0$-back & \\
\hline \multirow[t]{3}{*}{ González-Garrido et al., 2019} & 18 (7F) & Table 4 & $\mathrm{MNI}$ & A & $21.11(4.65)$ & Visual & Neutral Faces & 2-back $>$ rest & \\
\hline & 18 (7F) & Table 5 & $\mathrm{MNI}$ & A & $21.11(4.65)$ & Visual & Happy Faces & 2-back > rest & \\
\hline & 18 (7F) & Table 6 & $\mathrm{MNI}$ & A & $21.11(4.65)$ & Visual & Fear Faces & 2-back > rest & \\
\hline Goikolea et al. 2019 & $31(15 F)$ & $\begin{array}{l}\text { Table } 2 \\
\text { Table }\end{array}$ & Tal & $A / D$ & $31.06(8.76)$ & Visual & Letters & 2-back > baseline & Baseline $>2$-back \\
\hline Guimond et al. 2018 & $20(5 \mathrm{~F})$ & $1 \mathrm{~s}$ & $\mathrm{MNI}$ & $A / D$ & $25.05(4.05)$ & Visual & Faces & 2-back $>0$-back & 0-back $>$ 2-back \\
\hline Habel et al. 2007 & $22(\mathrm{OF})$ & Table 4 & $\mathrm{MNI}$ & A & $30.77(9.65)$ & Visual & Letters & 2-back $>0$-back & \\
\hline Harding et al. 2016 & $25(11 F)$ & Tabel 1 & $\mathrm{MNI}$ & A & $25.5(4.4)$ & Visual & Numbers & 2-back $>0$-back & \\
\hline Honey et al., 2000 & 20 (OF) & Table 1 & Tal & A & $39.3(13.6)$ & Visual & Letters & 2-back >0-back & \\
\hline Honey et al., 2003 & 27 (6F) & Table 3 & Tal & A & $35.1(9.9)$ & Visual & Letters & 2-back $>0$-back & \\
\hline Johannsen et al., 2013 & $12(8 \mathrm{~F})$ & Table 1 & $\mathrm{MNI}$ & A & $26.1(4.7)$ & Visual & Letters & 2-back >0-back & \\
\hline \multirow[t]{2}{*}{ Joseph et al., 2012} & $8(8 \mathrm{~F})$ & Table 1 & $\mathrm{MNI}$ & A & $25(6.4)$ & Visual & Letters & 2-back $>0$-back & \\
\hline & $8(8 F)$ & Table 1 & $\mathrm{MNI}$ & A & $25(6.4)$ & Visual & Letters & 2-back $>0$-back & \\
\hline Keresztes et al., 2004 & 29(20F) & Table 1 & $\mathrm{MNI}$ & A & $22.93(2.26)$ & Visual & Letters & 2-back $>0$-back & \\
\hline Kwon et al. 2001 & $15(15 F)$ & Table 3 & Tal & A & $15.05(4.58)$ & Visual & Letters & 2-back >0-back & \\
\hline Koppelstaetter et al., 2008 & $15(\mathrm{OF})$ & Table 2 & Tal & A & $25-47(5.58)$ & Visual & Letters & 2-back $>0$-back & \\
\hline
\end{tabular}




\begin{tabular}{|c|c|}
\hline Li et al., 2014 & $15(15 \mathrm{~F})$ \\
\hline Luo et al., 2014 & 25 (OF) \\
\hline \multirow[t]{2}{*}{ Lycke et al., 2008} & $26(14)$ \\
\hline & $26(14)$ \\
\hline Matsuo et al., 2007 & $15(10 \mathrm{~F})$ \\
\hline \multirow{2}{*}{ Meisenzahl et al., 2006} & $12(1 \mathrm{~F})$ \\
\hline & $12(1 \mathrm{~F})$ \\
\hline Monks et al., 2004 & 12 (OF) \\
\hline Oflaz et al., 2014 & $9(2 F)$ \\
\hline \multirow[t]{3}{*}{ Park et al., 2011} & 10 (OF) \\
\hline & 10 (OF) \\
\hline & 10 (OF) \\
\hline Paskavitz et al. 2010 & $17(9 F)$ \\
\hline Pfefferbaum et al., 2001 & $10(\mathrm{OF})$ \\
\hline Philip et al., 2016 & 13(9F) \\
\hline Quidé et al., 2013 & 28 (14F) \\
\hline \multirow{2}{*}{ Ragland et al., 2002} & $11(5 \mathrm{~F})$ \\
\hline & $11(5 \mathrm{~F})$ \\
\hline Rämä et al., 2001 & $8(8 \mathrm{~F})$ \\
\hline Reuter et al., 2008 & 49 (30F) \\
\hline \multirow[t]{2}{*}{ Rodriguez-Jimenez et al. 2009} & $13(6 \mathrm{~F})$ \\
\hline & $13(6 \mathrm{~F})$ \\
\hline \multirow[t]{2}{*}{ Rudner et al., 2013} & $20(15 \mathrm{~F})$ \\
\hline & $20(15 \mathrm{~F})$ \\
\hline Salavert et al., 2018 & $41(13 \mathrm{~F})$ \\
\hline Sanchez-Carrion et al., 2008 & $18(7 F)$ \\
\hline \multirow[t]{2}{*}{ Scheuerecker et al., 2008} & $23(4 \mathrm{~F})$ \\
\hline & $23(4 \mathrm{~F})$ \\
\hline \multirow[t]{2}{*}{ Schneiders et al., 2011} & $48(26)$ \\
\hline & $48(26)$ \\
\hline Seo et al., 2012 & $22(22 \mathrm{~F})$ \\
\hline Seo et al., 2014 & $34(34 \mathrm{~F})$ \\
\hline Stoodley et al., 2012 & 9 (OF) \\
\hline Stretton et al., 2012 & 15 (11F) \\
\hline
\end{tabular}

2-back > Rest

2 back $>$ rest

2 back $>$ rest

2-back $>0$-back

2-back $>0$-back

2 back $>0$ back (degraded)

2-back $>0$-back

2-back >0-back

\section{2-back > 0-back}

0-back > -back

2-back >0-back

2-back $>0$-back

2-back $>0$-back

2 back $>$ rest

0-back > 2-back

0-back > 2-back

rest > 2-back

2 back $>$ baseline

2-back > 0-back

2-back $>0$-back

2-back $>0$-back

2-back $>0$-back

2 back $>0$ back

2 back $>0$ back

2 back $>$ baseline (Phonological)

2 back $>$ baseline (Orthographic)
2 back $>$ baseline

2-back > 0 -Back

2-back >0-back

2-back degraded $>0$-back degraded

2 back $>0$ back pre-test

2 back $>0$ back post-test

2-back $>0$-back

0-back > 2-back

2-back $>0$-back

2-back > -back

0-back > 2-back

Location of dots 


\begin{tabular}{|c|c|c|c|c|c|c|c|c|c|}
\hline Suchan et al., 2005 & $13(8 \mathrm{~F})$ & Table 2 & Tal & A & 26 & Visual/auditory & Pictures & 2-back >0-back & \\
\hline Sumowski et al., 2010 & $18(15 \mathrm{~F})$ & Table 1 & Tal & $A / D$ & $43.8(7)$ & Visual & Letters & 2 back $>$ rest & rest > 2-back \\
\hline Sweet et al., 2010 & $12(7 F)$ & Table 1 & Tal & $A / D$ & $38.67(12.91)$ & Visual & Letters & 2-back $>0$-back & 0-back > 2-back \\
\hline Thermenos et al., 2011 & $10(5 F)$ & Tabel 2 & $\mathrm{MNI}$ & $A / D$ & $17.1(1.4)$ & Visual & Letters & 2-back >0-back & 0-back $>2$-back \\
\hline Thomas et al., 2005 & $16(1 \mathrm{~F})$ & Table 3 & Tal & A & $37.6(6.3)$ & Visual & Letters & 2-back >0-back & \\
\hline \multirow[t]{2}{*}{ Townsend et al., 2010} & $14(8 \mathrm{~F})$ & Table 3 & $\mathrm{MNI}$ & A & $30.8(6.0)$ & Visual & Letters & 2-back >0-back & \\
\hline & $14(8 \mathrm{~F})$ & Table 3 & $\mathrm{MNI}$ & A & $30.8(6.0)$ & Visual & Letters & 2-back $>0$-back & \\
\hline Valera et al., 2005 & $20(8 \mathrm{~F})$ & Table 3 & MNI & A & $33(10.6)$ & Visual & Letters & 2-back >0-back & \\
\hline Wu et al., 2017 & $45(21 F)$ & Table 2 & $\mathrm{MNI}$ & $A / D$ & $24.07(4.83)$ & Visual & Numbers & 2-back >0-back & 0 -back $>2$-back \\
\hline \multirow[t]{2}{*}{ Yan et al., 2011} & $28(16 \mathrm{~F})$ & Table 1 & Tal & $A / D$ & $20.4(1.4)$ & Visuospatial & White squares & 2-back>0-back (SL group) & 0-back $>2$-back \\
\hline & $28(16 \mathrm{~F})$ & Table 1 & Tal & $A / D$ & $20.9(1.5)$ & Visuospatial & White squares & 2-back> 0-back (HL group) & 0-back > 2-back \\
\hline Ziemus et al., 2008 & $9(4 \mathrm{~F})$ & Table2 & Tal & A & $44.2(9.6)$ & Visual & Letters & 2-back $>0$-back & \\
\hline
\end{tabular}

Notes: $A=$ Activation, $D=$ Deactivation

Table 2 - An overview of the cognitive reappraisal studies included in the meta-analysis

\begin{tabular}{|c|c|c|c|c|c|c|c|c|c|}
\hline Author & $\begin{array}{l}\mathrm{N} \\
\text { (Females) }\end{array}$ & Source & Space & $A / D$ & Age M (SD) & Stimuli & Instruction & Activation contrast & Deactivation contrast \\
\hline Albein-Urios et al. 2013 & 21(1F) & Table S2 & MNI & A & $31.00(4.60)$ & Negative and neutral pictures & Reappraise & Suppress>Maintain & \\
\hline Allard et al. 2014 & 34 (16F) & Table 2 & Tal & $A / D$ & $23.40(4.39)$ & Unpleasant film clips & Reappraise & Emotion regulation > Passive viewing & Passive viewing $>$ Emotion regulation \\
\hline Beauregard et al. 2001 & 10 (OF) & Table 2 & Tal & A & 23.5 & Erotic movies & Decrease/Distance & Attempted inhibition condition $>$ neutral & \\
\hline Campbell-Sills et al. 2011 & $26(22 \mathrm{~F})$ & Table 1 & Tal & A & $19.15(1.83)$ & Negative pictures & Reappraise & Reduce $>$ Maintain & \\
\hline Che, 2015 & 29(15F) & Table 1 & MNI & A & $22.62(1.59)$ & Negative pictures & Reappraise/decrease & Reduce $>$ maintain & \\
\hline Corbalan et al. 2015 & 17 (9F) & Table 3 / text & $\mathrm{MNI}$ & $A / D$ & $41.4(13.3)$ & Negative and neutral pictures & Reappraise & Decrease $>$ Look & Look > Decrease \\
\hline Cosme et al. 2018 & $33(16 \mathrm{~F})$ & Table 3 & $\mathrm{MNI}$ & $A / D$ & $18.12(0.34)$ & Food pictures & Reappraise & Regulate $>$ look & Look $>$ regulate \\
\hline de Wit et al. 2015 & 38 (20F) & Table 2 & MNI & A & $39.6(11.4)$ & Fear, OCD related, neutral pictures & Reappraise & Attend $>$ regulate & \\
\hline Delgado et al. 2008 & 12 (6F) & Table 2 & Tal & $A / D$ & $23.29(3.31)$ & Conditioned stimulus with shock & Reappraise & Regulate CS+>Attend CS+ & Attend versus Regulate CS+ Trials \\
\hline \multirow[t]{2}{*}{ Denny et al. 2015} & $21(11 \mathrm{~F})$ & Table S1 & $\mathrm{MNI}$ & A & $29(6.71)$ & Negative and neutral pictures & Reappraise & Reappraise Cue > Look Cue & \\
\hline & 21 (11F) & Table S3 & MNI & A & $29(6.71)$ & Negative and neutral pictures & Reappraise & Reappraise Negative $>$ Look Negative & \\
\hline Domes et al. 2010 & 33 (17F) & Table IV & MNI & A & $m: 25.2(1.9) f: 24.6(1.6)$ & Negative pictures & Reappraise & Decrease $>$ Maintain & \\
\hline Eippert et al. 2007 & 24 (24F) & Table II, III & MNI & $A / D$ & 23.3 & Negative pictures & Reappraise/distance & Decrease > View & \\
\hline \multirow[t]{2}{*}{ Erk et al. 2010} & $17(8 \mathrm{~F})$ & Table 2 & MNI & $A / D$ & $43.9(10.1)$ & Negative and neutral pictures & Reappraise & Regulation $>$ No regulation & Negative no regulation $>$ regulation \\
\hline & $17(8 \mathrm{~F})$ & Table 2 & MNI & $A / D$ & $43.9(10.1)$ & Negative and neutral pictures & Reappraise & & Negative no regulation $>$ regulation \\
\hline Fitzgerald et al. 2018 & 49 (67\%F) & Table 2 & MNI & A & $25.24(7.98)$ & Negative and neutral pictures & Reappraise & Reappraise>Look-Negative & \\
\hline Giuliani et al. 2014 & $55(33 \mathrm{~F})$ & Table 1 & $\mathrm{MNI}$ & $A / D$ & $22.17(2.36)$ & Food pictures & Reappraise & Regulate $>$ Look & Look $>$ Regulate \\
\hline Goldin et al. 2008 & 17 (17F) & Table 2 & Tal & A & $22.7(3.5)$ & Negative film clips & Reappraise & Reappraise $>$ Watch-Negative (Early) & \\
\hline Goldin et al. 2019 & $35(20 F)$ & Table 3 & Tal & $A / D$ & $32.2(8.9)$ & Autobiographical social situations & Reappraise & Reappraisal $>$ React & React $>$ Reappraisal \\
\hline Golkar et al. 2012 & $58(32 \mathrm{~F})$ & Table S1 & MNI & A & $24.02(2.26)$ & Negative and neutral pictures & Reappraise & Reappraise > Attend & \\
\hline Grecucci et al. 2012 & 21 (10F) & Table 2 & MNI & A & $23.5(3.6)$ & Ultimate game / unfair offers & Reappraise & Unfair accepted Down > Look & \\
\hline
\end{tabular}




\begin{tabular}{|c|c|c|c|c|c|c|c|c|c|}
\hline Hallam et al. 2015 & 20 (?F) & Table 3 & Tal & $A / D$ & 20 (?) & Negative and neutral pictures & Reappraise & Implementation intention > goal intention & Goal intention > Implementation intention \\
\hline \multirow[t]{2}{*}{ Harenski and Hamman 2006} & 10 (10F) & Table 3 & MNI & A & $18-29$ & Moral and non-moral pictures & Reappraise & Decrease moral > odd-even baseline & \\
\hline & 10 (10F) & Table 3 & MNI & A & $18-29$ & Moral and non-moral pictures & Reappraise & Decrease non-moral > odd-even baseline & \\
\hline Hayes et al. 2010 & 25 (11F) & Table 1,2 & MNI & $\mathrm{A} / \mathrm{D}$ & $21.6(2.5)$ & Negative pictures & Reappraise & Reappraise > View & View > reappriase \\
\hline Hollmann et al. 2012 & $17(17 \mathrm{~F})$ & Table 1 & MNI & A & $25.3(3.1)$ & High-caloric food pictues & Reappraise & Regulate_tasty versus Admit_tasty & \\
\hline Kanske et al. 2011 & 30 (17F) & Table 3 & MNI & $A / D$ & $21.8(2.1)$ & Negative and positive pictures & Reappraise & Reappraisal--view emotional & View emotional--reappraisal \\
\hline \multirow[t]{2}{*}{ Kanske et al. 2012} & 25 (18F) & ST2 & $\mathrm{MNI}$ & $A / D$ & $43.88(11.21)$ & Negative and positive pictures & Reappraise & reappraisal positive - view positive & view positive - reappraisal positive \\
\hline & $25(18 \mathrm{~F})$ & ST2 & MNI & $A / D$ & $43.88(11.21)$ & Negative and positive pictures & Reappraise & reappraisal negative - view negative & view negative - reappraisal negative \\
\hline \multirow[t]{2}{*}{ Kim and Hamann 2007} & 10 (10F) & Table 3 & MNI & A & 20.7 & Negative and positive pictures & Reappraise & Decrease $>$ Watch Contrast for Negative Pictures & \\
\hline & 10 (10F) & Table 4 & MNI & A & 20.7 & Negative and positive pictures & Reappraise & Decrease $>$ Watch Contrast for Positive Pictures & \\
\hline Koenigsberg et al., 2010 & $16(9 F)$ & Table 1 & MNI & $A / D$ & $31.8(7.7)$ & Negative and neutral pictures & Reappraise/distance & Distancing $\geq$ looking & Looking $\geq$ distancing \\
\hline Korb et al. 2015 & 18 (10F) & Table 3 & MNI & $A / D$ & 27 & Angry prosody & Reappraise & Decrease > Feel Negative & Feel Negative > Decrease \\
\hline Krendl et al. 2012 & 20 (10F) & Table 1 & MNI & $A / D$ & 21.6 & (Non)stigmatized negative pictures & Reappraise & Decrease IAPS > attend IAPS & Attend IAPS > decrease IAPS \\
\hline Lang et al. 2012 & $15(15 F)$ & Table S3 & $\mathrm{MNI}$ & A & $24.73(5.64)$ & Negative and neutral scripts & Reappraise/distance & Down vs. maintain & \\
\hline Leiberg et al. 2012 & $24(24 \mathrm{~F})$ & Table S2 & MNI & $\mathrm{A} / \mathrm{D}$ & 24.1 & Negative and neutral pictures & Reappraise/distance & Disengage-minus-view & View-minus-Disengage \\
\hline \multirow[t]{2}{*}{ Mak et al. 2009} & $12(12 \mathrm{~F})$ & Table 1 & MNI & $A / D$ & $24(1.78)$ & Positive pictures & Reappraise & Regulate > view & View $>$ Regulate \\
\hline & $12(12 \mathrm{~F})$ & Table 1 & MNI & $A / D$ & $24(1.78)$ & Negative pictures & Reappraise & Regulate $>$ view & View $>$ Regulate \\
\hline McRae et al. 2010 & 18 & Tabel 3 & $\mathrm{MNI}$ & A & $24.4(3.5)$ & Negative and neutral pictures & Reappraise & Reappraise $>$ Look & \\
\hline McRae et al. 2008 & $25(13 \mathrm{~F})$ & Table 1 & MNI & A & $\mathrm{m}: 20.36 \mathrm{f}: 20.6$ & Negative pictures & Reappraise & Decrease Negative > Look Negative & \\
\hline Modinos et al. 2010 & $18(7 F)$ & Table 1 & MNI & A & $21.1(2.8)$ & Negative and neutral pictures & Reappraise & Reappraisal > Negative & \\
\hline \multirow[t]{2}{*}{ Moodie et al. 2020} & 30(17F) & Table 2 & $\mathrm{MNI}$ & A & 24.3 & Negative and neutral pictures & Reappraise & Reappraisal > Watch (Low) & Watch > Reappraisal (Low) \\
\hline & 30(17F) & Table 2 & $\mathrm{MNI}$ & A & 24.3 & Negative and neutral pictures & Reappraise & Reappraisal > Watch (High) & Watch > Reappraisal (High) \\
\hline Morawetz et al. 2017 & $23(12 \mathrm{~F})$ & Table 2 & Tal & $A / D$ & $25.70(5.95)$ & Negative and neutral pictures & Reappraise/distance & Decrease > Look Negative & Look Negative > Decrease \\
\hline Nelson et al. 2015 & $22(11 \mathrm{~F})$ & Table 1 & $\mathrm{MNI}$ & A & $25.2(5.8)$ & Negative and neutral pictures & Reappraise & Reappraise > Maintain & \\
\hline New et al. 2009 & $14(14 F)$ & Table S3 & $\mathrm{MNI}$ & A & $31.7(10.3)$ & Negative pictures & Reappraise & Diminish minus maintain & \\
\hline Ochsner et al. 2002 & $15(15 F)$ & Table 1,2 & MNI & $A / D$ & 21.9 & Negative and neutral pictures & Reappraise & Reappraise > Attend & Attend > Reappraise \\
\hline Ochsner et al. 2004 & $24(24 \mathrm{~F})$ & Table 2,3 & MNI & $A / D$ & 20.6 & Negative pictures & Reappraise & Decrease > Look & Look > Decrease \\
\hline Otto et al. 2014 & $26(26 \mathrm{~F})$ & Table 1 & Tal & $A / D$ & $24.9(5.6)$ & Fearful faces + emotional information & Reappraise & reappraise versus look & look versus reappraise \\
\hline Paret et al. 2011 & 21 (OF) & Table 1 & MNI & A & $28(4)$ & Shock or no shock & Reappraise & Main effect of reappraisal (R-NR) & \\
\hline Paschke et al. 2016 & $108(55 F)$ & $\begin{array}{l}\text { Table S5 + } \\
\text { text }\end{array}$ & MNI & $A / D$ & $26.12(3.7)$ & Negative and neutral pictures & Distance & RegulateNeg $>$ WatchNeg & WatchNeg>RegulateNeg \\
\hline Phan et al. (2005) & $14(8)$ & Table 1 & MNI & $A / D$ & $27.6(4.4)$ & Negative pictures & Reappraise & $\mathrm{s}>\mathrm{M}$ & $M>S$ \\
\hline Price et al. 2013 & $11(8 \mathrm{~F})$ & Table 2 & Tal & $A / D$ & $22.2(2.2)$ & Autobiographical Memories & Reappraise & Reappraisal > fixation & Fixation > reappraisal \\
\hline \multirow[t]{2}{*}{ Qu et al. 2017} & 29 (14F) & Table 1 & MNI & A & 19.2 & Negative pictures & Reappraise & decrease-look (positive activation) & \\
\hline & 29 (14F) & Table 1 & MNI & A & 19.2 & Negative pictures & Reappraise & decrease-look (negative activation) & \\
\hline Sarkheil et al. 2015 & $14(8 \mathrm{~F})$ & Table 2 & Tal & $A / D$ & range $20-27$ & Negative pictures & Reappraise & Reappraisal > view & View $>$ reappraisal \\
\hline Schardt et al. 2010 & 37 (37F) & Table 1 & MNI & $A / D$ & $22.6(2.2)$ & Fear, disgust, neural pictures & Reappraise & Regulation > perception & Perception>Regulation \\
\hline Schienle et al. 2017 & $45 \mathrm{~F}$ & Table 1 & MNI & A & $22.91(3.21)$ & Disgusting and neutral pictres & Reappraise & Reappraisal > Passive Viewing & \\
\hline Schulze et al. 2011 & $15 \mathrm{~F}$ & Table S2 & MNI & $A / D$ & $24.53(2.85)$ & Negative and neutral pictures & Reappraise & decrease > maintain emotions $\mathrm{HC}$ & maintain > decrease emotions $\mathrm{HC}$ \\
\hline Shermohammed et al. 2017 & 25(12F) & Table 3 & $\mathrm{MNI}$ & A & $20.89(1.71)$ & Negative pictures & Reappraise & decrease-negative > look-negative & \\
\hline Silvers et al. 2015 & $30(13 \mathrm{~F})$ & Table 1 & $\mathrm{MNI}$ & A & 21.97 & Negative and neutral pictures & Reappraise & Reappraise/low>Look/low & \\
\hline
\end{tabular}




\begin{tabular}{|c|c|c|c|c|c|c|c|c|c|}
\hline & $30(13 F)$ & Table 1 & MNI & A & 21.97 & Negative and neutral pictures & Reappraise & Reappraise/high>Look/high & \\
\hline Simsek et al. 2017 & 15 & Table 3 & MNI & A & $22.53(1.80)$ & Negative and neutral pictures & Reappraise & Reappraise Neg > Attend Negative & \\
\hline \multirow[t]{2}{*}{ Sokol-Hessner et al. (2013) } & 16 & Table 1 & Tal & A & $19.8(3.1)$ & Monetary decisions & Reappraise & Regulate decision ME > Attend decision ME & \\
\hline & 14 & Table S3 & Tal & $A / D$ & 19.8(3.1) & Monetary decisions & Reappraise & Regulate Lose ME > Attend Lose ME & Attend Lose ME > Regulate Lose ME \\
\hline Sripada et al. 2014 & $49(23 \mathrm{~F})$ & Table 3 & MNI & $A / D$ & $23.63(1.3)$ & aversive or neutral pictures & reappraise & Reappraise > Maintain & Maintain > Reappraise \\
\hline Staudinger et al. 2009 & $16(8 \mathrm{~F})$ & In text & MNI & A & $23.1(3.1)$ & Reward anticipation & Reappraise/distance & Distance vs. Permit & \\
\hline Staudinger et al. 2011 & $24(13 F)$ & Table 1 & MNI & A & $25.1(2.8)$ & Reward anticipation & Reappraise/distance & Regulate $>$ permit & \\
\hline Van der Meer et al. 2014 & $20(6 \mathrm{~F})$ & Table 3 & MNI & A & $35.5(11.7)$ & Negative and neutral pictures & Reappraise & Reappraise > Attend negative HC & \\
\hline Van der Velde et al. 2015 & 51 (?F) & Table S1 & MNI & A & $37.1(10.3)$ & Negative and neutral pictures & reappraise & Reappraise > Attend negative & \\
\hline Van der Velde et al. 2015 & $16(8 \mathrm{~F})$ & Table S1 & MNI & A & $22.1(3.6)$ & Negative and neutral pictures & Reappraise & Reappraise > Attend negative HC & \\
\hline Vanderhasselt et al. 2013 & $42(42 F)$ & Table 1 & MNI & A & $21.26(2.29)$ & Negative pictures & Reappraise & Target Reappraisal > Target Appraise & \\
\hline Walter et al. 2009 & $18(18 \mathrm{~F})$ & Table 1 & MNI & $A / D$ & $24(3)$ & Negative and neutral pictures & Reappraise & Regulation $>$ no regulation & No regulation > regulation \\
\hline \multirow[t]{2}{*}{ Winecoff et al. 2013} & $31(21 \mathrm{~F})$ & Tabe 1 & MNI & A & 25 & Negative and positive pictures & Reappraise & Negative Regulate > Negative Experience $(\exp 1)$ & \\
\hline & $31(21 \mathrm{~F})$ & Tabe 1 & MNI & $A / D$ & 25 & Negative and positive pictures & Reappraise & Positive Regulate > Positive Experience (exp1) & Positive Experience > Positive Regulate \\
\hline \multirow[t]{2}{*}{ Ziv et al. 2013} & $27(13 F)$ & Table 2 & Tal & A & $32.6(9.5)$ & Pictures of faces & Reappraise & HC only: Reappraise > React (faces task) & \\
\hline & $27(13 F)$ & Table 3 & Tal & A & $32.6(9.5)$ & Pictures of faces & Reappraise & HC only: Reappraise > React (criticism task) & \\
\hline
\end{tabular}

Notes: $A=$ Activation, $D=$ Deactivation 


\section{The ALE meta-analysis procedure}

We performed the meta-analysis using the activation likelihood estimation (ALE) algorithm implemented in the software GingerALE version 3.0.2 (http://www.brainmap.org/ale; Eickhoff et al., 2012, 2009; Turkeltaub et al., 2012). ALE is a coordinate-based method used for performing meta-analyses of human brain imaging studies. A Full-Width Half-Maximum (FWHM) of the Gaussian function is used to blur the foci. The size of the gaussian is determined by the number of subjects in each experiment. An ALE image is created based on all coordinates. Significance is determined via a permutation procedure which we set to 1000 permutations. We used a cluster-forming voxel-level threshold of $p<0.001$ (uncorrected). Alpha was set at 0.05, whole-brain family-wise error (FWE) corrected at the cluster level. Before the analysis, we converted all coordinates in Talairach space to MNI space using the GingerALE foci converter tool. The analyses were done on the MNI coordinates.

In addition, we performed a comparison analysis on the deactivation contrasts (Control > 2-back and Control > Reappraise) including a conjunction and subtraction analysis. In the conjunction analysis, a conjunction image was created using the voxel-wise minimum value of the two contrast (Control > 2-back and Control > Reappraise) ALE maps. The conjunction output image shows the similarity in clusters between the two contrast maps. In the subtraction analysis, two contrast (Control > 2-back and Control > Reappraise) ALE maps are directly subtracted from each other. In addition, we performed a "pooled" analysis following the procedure described above, including the coordinates from both contrasts. The pooled data was subsequently used for permutation testing where the data was randomly assigned to one of the two contrasts and repeated 10,000 times. The subtraction maps were tested against this null distribution.

Anatomical labels provided by the GingerALE software are derived from the Talairach Daemon atlas (talairach.org). For the amygdala deactivation clusters, we reported the \% of that cluster falling in the amygdala based on those labels. 


\section{Results}

\section{ALE meta-analysis activation contrasts}

We first verified regions that were systematically activated during a 2-back working memory task or a cognitive reappraisal task compared to a control task (i.e., 2-back > Control and Reappraisal > Control). We found 10 clusters for the 2-back > Control contrast among which are located in the left [cluster $\# 1, z=9.34, p=4.95 E-21, m m 3=23680$ ] and right [cluster \#2, $z=8.44, p=6.53 E-18, m m 3=18840$ ] dorsolateral prefrontal cortex $($ dIPFC $)$, the left [cluster \#3, $z=10.32, p=2.69 E-25, \mathrm{~mm} 3=12440$ ] and right [cluster $\# 4, z=9.92, p=2.36 E-19, m m 3=12296$ ] posterior parietal cortex (PPC), the left [cluster $\# 1, z=9.34, p=4.95 E-21, \mathrm{~mm} 3=23680$ ] and right [cluster \#2, z=8.44, $\mathrm{p}=6.53 \mathrm{E}-18, \mathrm{~mm} 3=18840$ and cluster \#6, $\mathrm{z}=12.84, \mathrm{p}=4.82 \mathrm{E}-38$, $\mathrm{mm} 3=5104$ ] anterior insula, and the left/right [cluster \#5, $z=8.03, p=5.03 E-16, \mathrm{~mm} 3=9032$ ] dorsal anterior cingulate cortex (dACC). See Table 3 for a full overview of the clusters and statistics.

We found 9 clusters for the Reappraisal > Control contrast among which are the left [cluster \#3, $z=6.91, p=2.46 \mathrm{E}-12, \mathrm{~mm} 3=7320$ ] and right [cluster \#8, $\mathrm{z}=5.61, \mathrm{p}=1.01 \mathrm{E}-08$, $\mathrm{mm} 3=2.46 \mathrm{E}-12$ ] dorsolateral prefrontal cortex (dIPFC), the left [cluster $\# 2, z=8.10, p=2.81 \mathrm{E}-$ $16, \mathrm{~mm} 3=9136$ ] and right [cluster \#4, $\mathrm{z}=8.44, \mathrm{p}=6.53 \mathrm{E}-18, \mathrm{~mm} 3=18840$ and cluster \#6, $z=7.69, p=7.60 E-15, \mathrm{~mm} 3=6552$ ] anterior insula, and the left/right [cluster $\# 1, z=8.80, p=$ $6.66 \mathrm{E}-19, \mathrm{~mm} 3=10880$ ] dorsal anterior cingulate cortex (dACC). See Table 3 for a full overview of the clusters and statistics.

Together these findings are in line with previous meta-analyses' reports of activation patterns during working memory (Wager and Smith, 2003), a 2-back working memory task (Lee and Xue, 2018), and a cognitive reappraisal task (Buhle et al., 2014; Kohn et al., 2014; Lee and Xue, 2018).

\section{ALE meta-analysis deactivation contrasts}

The main aim of this study was to investigate whether the amygdala is systematically downregulated during working memory in a similar fashion as it is during emotion regulation.

Indeed, for the Control > 2-Back working memory contrast we saw clusters in the left [cluster \#3, z=5.53, p= 1.56E-08, mm3=1952] and right [cluster \#4, $z=5.70, p=6.16 \mathrm{E}-09$, $\mathrm{mm} 3=1160$ ] amygdala. These clusters fall for $82.6 \%$ within the left amygdala and $91.5 \%$ within 
the right amygdala. We also observed a cluster in left/right [cluster \#2, z=6.18, p=3.27E-10, $\mathrm{mm} 3=5480$ ] ventral medial prefrontal cortex (vmPFC), and the left/right [cluster \#1, $z=6.63$ $, p=1.68 E-11, m m 3=5568]$ posterior cingulate cortex (PCC). See Figure 2 and Table 4.

For the Control > Reappraisal contrast we also observed clusters in the left [cluster \#2, $z=9.02, p=9.55 E-20, m m 3=2992$ ] and right [cluster $\# 1, z=7.45, p=4.70 E-14, m m 3=3728$ ] amygdala, [cluster \#3, z=5.75, $\mathrm{p}=4.55 \mathrm{E}-09, \mathrm{mm3}=952$ ] which overlap with the amygdala clusters found during the Control > 2-Back contrast. These clusters falls for $75.8 \%$ within the left amygdala and 59.2\% within the right amygdala. See Figure 2 and Table 4.

In sum, there is reduced amygdala activity during cognitive reappraisal compared to a control task, as has been shown before (Buhle et al., 2014). Critically, this is also the case during a 2-back working memory task compared to a control task.

\section{Comparison analysis of the deactivation contrasts}

Finally, we performed a comparison analysis between the deactivation contrasts (Control > 2-back and Control > Reappraise). The conjunction analysis revealed there is an overlap in deactivation patterns during cognitive reappraisal and the 2-back working memory task in the amygdala [left: $96.7 \%$ falls within the amygdala, right: $91.1 \%$ falls within the amygdala]. The subtraction analysis revealed that a cluster partly falling within the amygdala [left: $55 \%$ falls within the amygdala, 30\% falls in the dorsal entorhinal cortex (BA34)] was present stronger for cognitive reappraisal compared to the 2-back working memory task, and a cluster partly falling within the amygdala [left: $5 \%$ falls in the amygdala, $90 \%$ falls in the hippocampus] was present for the 2-back working memory task compared to cognitive reappraisal.

In sum, both 2-back working memory and cognitive reappraisal tasks show bilateral clusters of common deactivation in the amygdala. The deactivation clusters associated with both tasks do differ somewhat in their topography, with stronger deactivation extending from (left) amygdala toward entorhinal cortex for cognitive reappraisal, and toward hippocampus for working memory. See Figure 3 and Table 5. 


\section{ALE meta-analysis: deactivation}
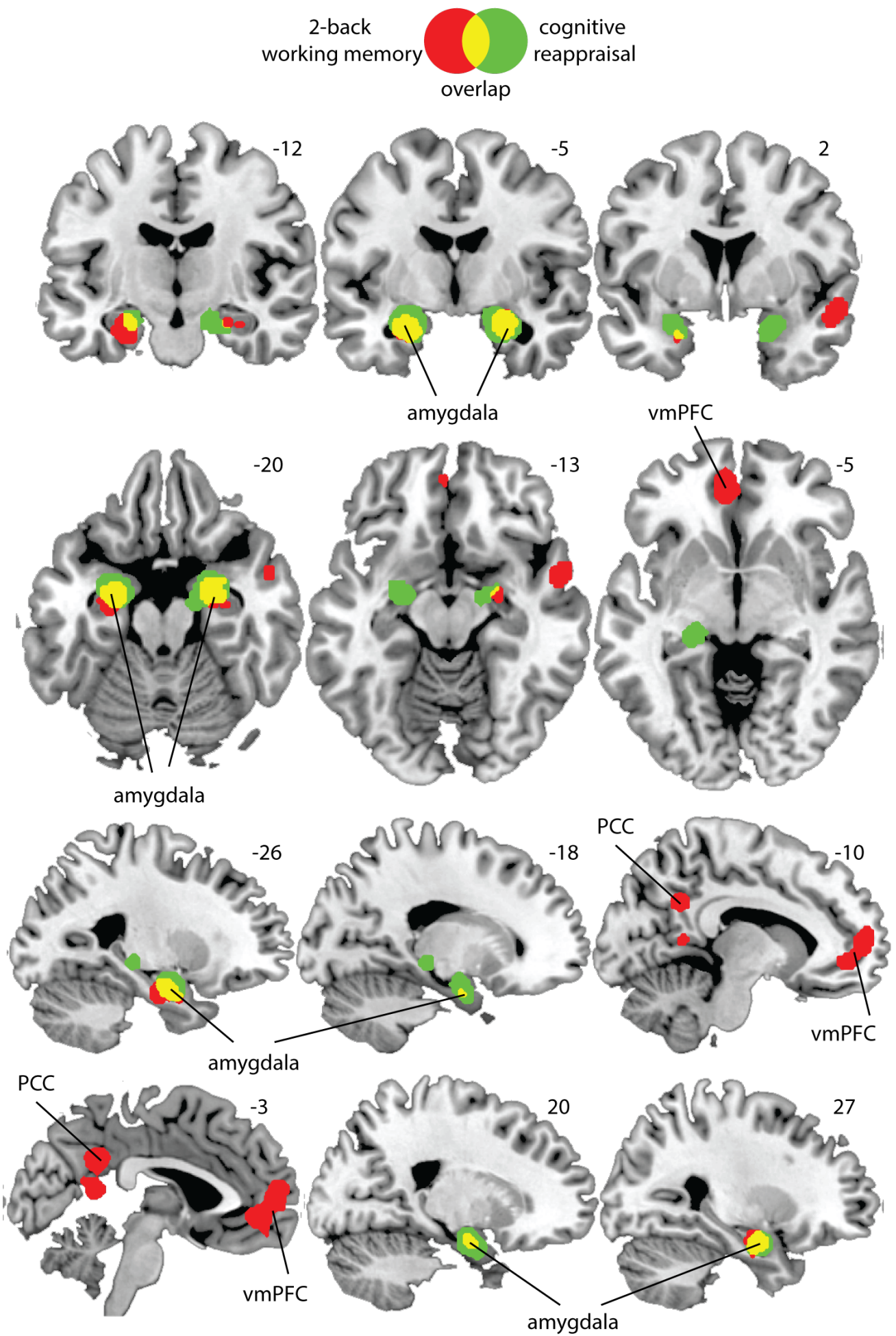

Figure 2 - Display of the significant clusters for the ALE meta-analysis on the deactivation contrasts Control > 2-Back (red) and Control > Cognitive reappraisal (green) and the overlap (yellow). 


\section{ALE meta-analysis: deactivation

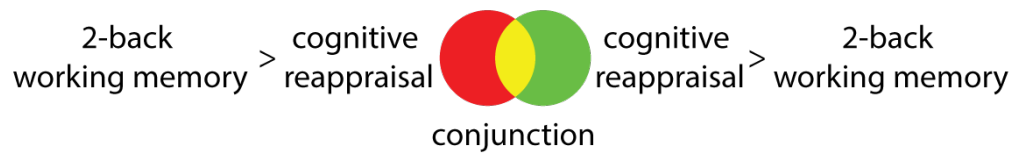
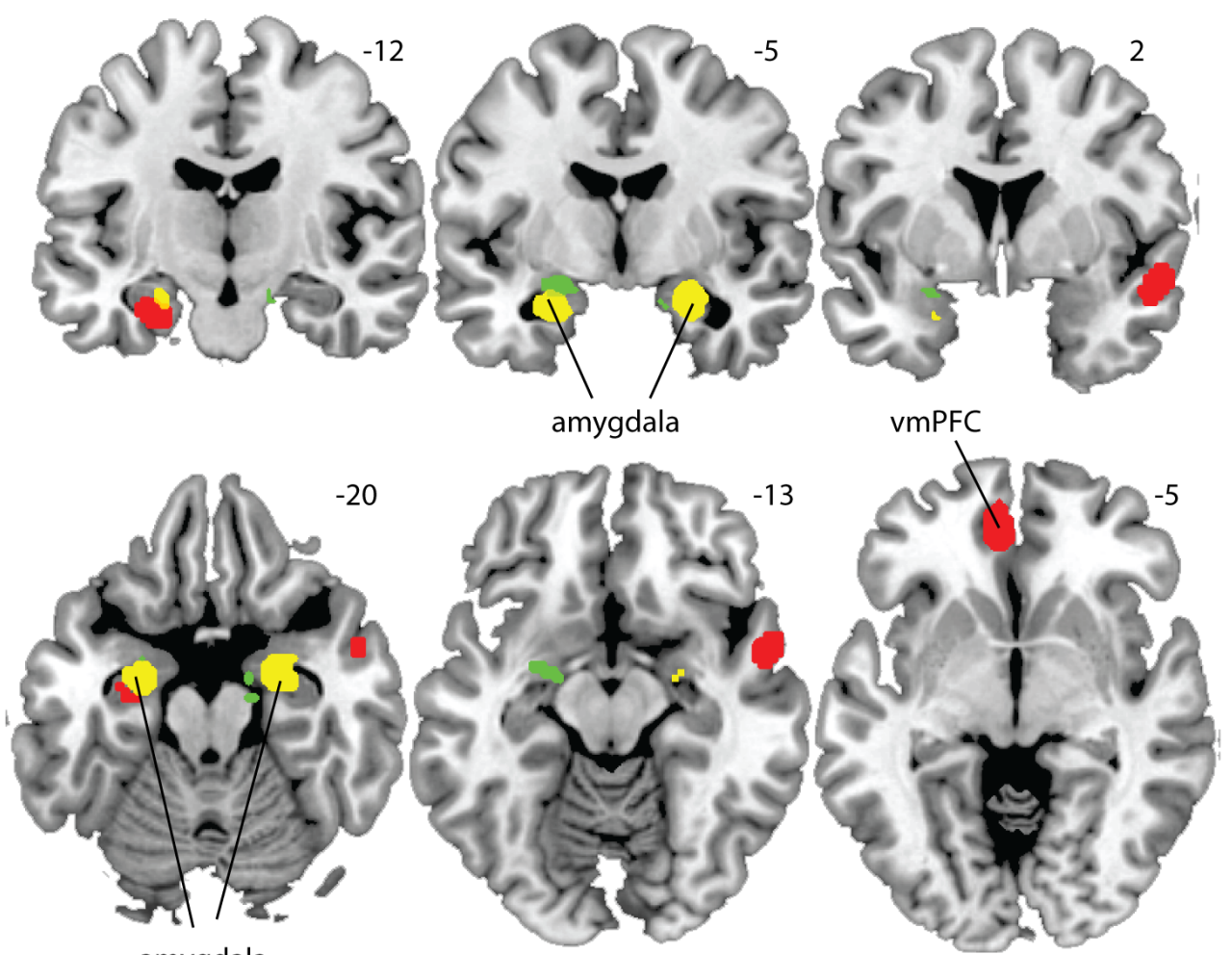

amygdala
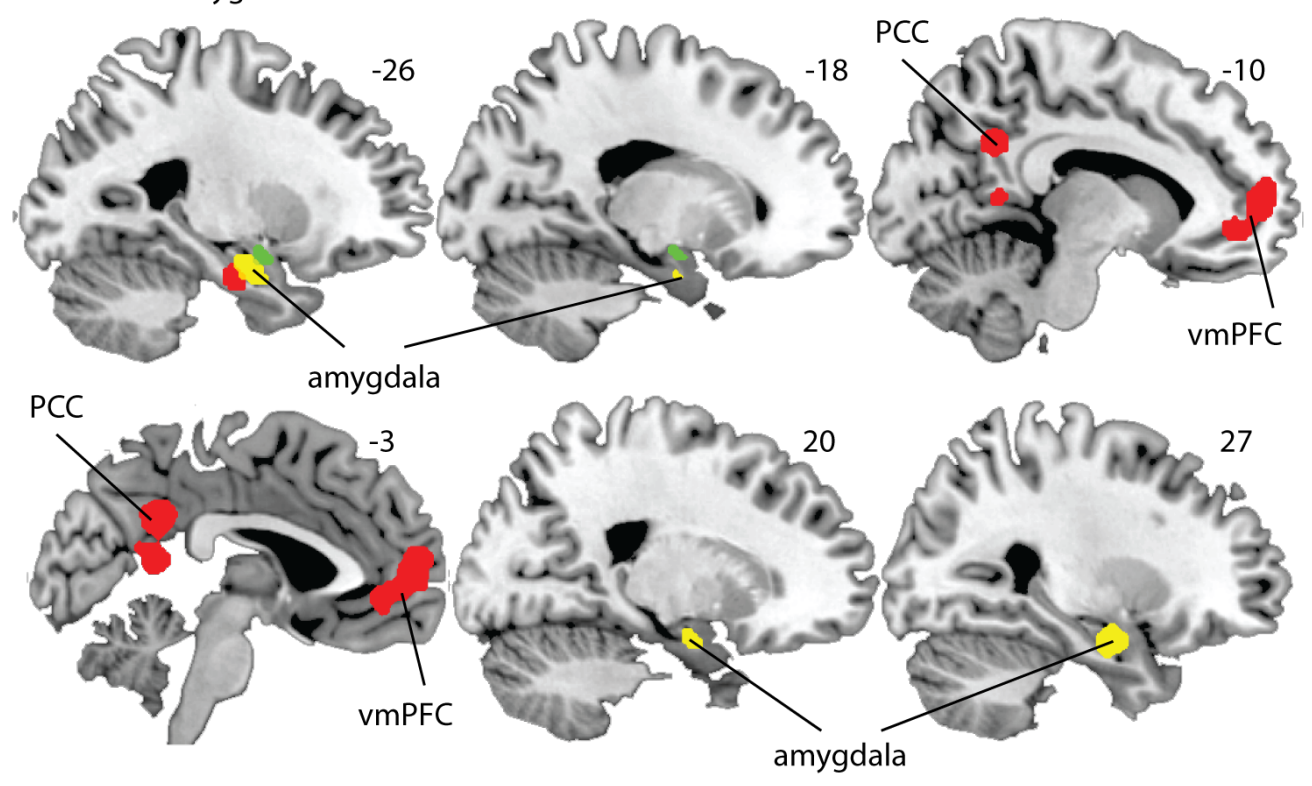

Figure 3 - Display of the significant clusters for the ALE meta-analysis on the differences in deactivation contrasts Control > 2-Back (red) and Control > Cognitive reappraisal (green) and the conjunction of the two where the show common activation (yellow). 
Table 3 - Significant clusters from the ALE meta-analysis showing an activation pattern

\begin{tabular}{|c|c|c|c|c|c|c|c|c|c|}
\hline Cluster \# & Region & Side & $X(\mathrm{~mm})$ & $Y(m m)$ & $\mathrm{Z}(\mathrm{mm})$ & $\mathrm{mm}^{\wedge} \mathbf{3}$ & ALE & $\mathbf{P}$ & Peak Z \\
\hline \multicolumn{10}{|c|}{ WM activation (2-back $>$ Control) } \\
\hline \multirow[t]{6}{*}{$\# 1$} & anterior insula / dorsolateral prefrontal cortex (dIPFC) & L & -32 & 22 & 0 & 23680 & 0.078 & $4.95 \mathrm{E}-21$ & 9.34 \\
\hline & & & -42 & 8 & 30 & & 0.076 & $3.56 \mathrm{E}-20$ & 9.13 \\
\hline & & & -40 & -8 & 40 & & 0.046 & $6.95 \mathrm{E}-11$ & 6.42 \\
\hline & & & -28 & -2 & 52 & & 0.043 & 5.97E-10 & 6.08 \\
\hline & & & -30 & -8 & 48 & & 0.039 & $7.72 \mathrm{E}-09$ & 5.66 \\
\hline & & & -36 & 38 & 24 & & 0.034 & $1.90 \mathrm{E}-07$ & 5.08 \\
\hline \multirow[t]{8}{*}{ \#2 } & anterior insula / dorsolateral prefrontal cortex (dIPFC) & $\mathrm{R}$ & 30 & 6 & 58 & 18840 & 0.069 & $6.53 \mathrm{E}-18$ & 8.54 \\
\hline & & & 40 & 28 & 30 & & 0.062 & $1.11 \mathrm{E}-15$ & 7.93 \\
\hline & & & 30 & -2 & 48 & & 0.057 & $6.43 E-14$ & 7.41 \\
\hline & & & 32 & 38 & 22 & & 0.042 & 1.63E-09 & 5.92 \\
\hline & & & 32 & 46 & 20 & & 0.039 & $1.08 \mathrm{E}-08$ & 5.60 \\
\hline & & & 36 & 6 & 32 & & 0.039 & $1.09 \mathrm{E}-08$ & 5.60 \\
\hline & & & 44 & 12 & 26 & & 0.029 & 2.67E-06 & 4.55 \\
\hline & & & 22 & -12 & 58 & & 0.020 & $4.90 \mathrm{E}-04$ & 3.30 \\
\hline \multirow[t]{4}{*}{ \#3 } & posterior parietal cortex / angular gyrus & $\mathrm{L}$ & -42 & -44 & 42 & 12440 & 0.090 & $2.69 \mathrm{E}-25$ & 10.33 \\
\hline & & & -28 & -60 & 38 & & 0.077 & $1.38 \mathrm{E}-20$ & 9.23 \\
\hline & & & -34 & -54 & 46 & & 0.070 & $2.27 \mathrm{E}-18$ & 8.66 \\
\hline & & & -20 & -70 & 54 & & 0.021 & $2.58 \mathrm{E}-04$ & 3.47 \\
\hline \multirow[t]{2}{*}{ \#4 } & posterior parietal cortex / angular gyrus & $\mathrm{R}$ & 30 & -62 & 44 & 12296 & 0.073 & $2.36 \mathrm{E}-19$ & 8.92 \\
\hline & & & 40 & -46 & 42 & & 0.071 & $1.07 \mathrm{E}-18$ & 8.75 \\
\hline \multirow[t]{2}{*}{ \#5 } & dorsal anterior cingulate cortex & $L / R$ & -2 & 8 & 50 & 9032 & 0.063 & 5.03E-16 & 8.03 \\
\hline & & & 8 & 26 & 32 & & 0.029 & $3.54 \mathrm{E}-06$ & 4.49 \\
\hline \#6 & anterior insula & $\mathrm{R}$ & 32 & 22 & -2 & 5104 & 0.123 & $4.82 E-38$ & 12.84 \\
\hline \multirow[t]{3}{*}{ \#7 } & cerebellum & & 30 & -62 & -32 & 3688 & 0.036 & $6.64 \mathrm{E}-08$ & 5.28 \\
\hline & & & 26 & -60 & -20 & & 0.030 & 1.49E-06 & 4.67 \\
\hline & & & 40 & -62 & -18 & & 0.027 & $9.14 \mathrm{E}-06$ & 4.28 \\
\hline \multirow[t]{2}{*}{ \#8 } & fusiform gyrus & $\mathrm{L}$ & -40 & -60 & -18 & 2240 & 0.032 & 7.37E-07 & 4.81 \\
\hline & & & -32 & -64 & -30 & & 0.027 & 1.07E-05 & 4.25 \\
\hline \#9 & caudate / putamen & $\mathrm{L}$ & -16 & -2 & 16 & 1464 & 0.034 & $1.53 \mathrm{E}-07$ & 5.12 \\
\hline$\# 10$ & middle frontal gyrus & L & -36 & 56 & 14 & 1456 & 0.037 & $3.24 \mathrm{E}-08$ & 5.41 \\
\hline \multicolumn{10}{|c|}{ CR activation (Reappraisal > Control) } \\
\hline \multirow[t]{3}{*}{$\# 1$} & dorsal anterior cingulate cortex & $L / R$ & -6 & 14 & 62 & 10880 & 0.072 & $6.66 \mathrm{E}-19$ & 8.80 \\
\hline & & & 12 & 18 & 62 & & 0.037 & $2.28 \mathrm{E}-08$ & 5.47 \\
\hline & & & 4 & 28 & 40 & & 0.031 & $7.13 E-07$ & 4.82 \\
\hline
\end{tabular}


\#2 anterior insula

\#3 dorsolateral prefrontal cortex (dIPFC)

\#4 anterior insula

\#5 middle temporal gyrus / angular gyrus

\#6 middle temporal gyrus

\#7 angular gyrus

\#8 dorsolateral prefrontal cortex (dIPFC)
20

\#9 middle cingulate cortex

\begin{tabular}{rrrrrr}
12 & 60 & & 0.029 & $2.46 \mathrm{E}-06$ & 4.57 \\
24 & 44 & & 0.024 & $2.70 \mathrm{E}-05$ & 4.04 \\
36 & 38 & & 0.024 & $3.30 \mathrm{E}-05$ & 3.99 \\
20 & 46 & & 0.022 & $1.06 \mathrm{E}-04$ & 3.70 \\
28 & -8 & 9136 & 0.063 & $2.81 \mathrm{E}-16$ & 8.10 \\
22 & -2 & & 0.045 & $1.06 \mathrm{E}-10$ & 6.35 \\
46 & -6 & & 0.037 & $1.92 \mathrm{E}-08$ & 5.50 \\
6 & 48 & 7320 & 0.051 & $2.46 \mathrm{E}-12$ & 6.91 \\
20 & 46 & & 0.039 & $5.25 \mathrm{E}-09$ & 5.72 \\
30 & -8 & 6552 & 0.059 & $7.60 \mathrm{E}-15$ & 7.69 \\
44 & -10 & & 0.035 & $4.38 \mathrm{E}-08$ & 5.35 \\
18 & -4 & & 0.034 & $9.59 \mathrm{E}-08$ & 5.21 \\
24 & 6 & & 0.028 & $3.94 \mathrm{E}-06$ & 4.47 \\
22 & -12 & & 0.021 & $1.59 \mathrm{E}-04$ & 3.60 \\
-56 & 22 & 5488 & 0.039 & $5.08 \mathrm{E}-09$ & 5.73 \\
-52 & 44 & & 0.036 & $4.20 \mathrm{E}-08$ & 5.36 \\
-64 & 42 & & 0.034 & $8.50 \mathrm{E}-08$ & 5.23 \\
-62 & 34 & & 0.032 & $3.04 \mathrm{E}-07$ & 4.99 \\
-52 & 20 & & 0.023 & $7.03 \mathrm{E}-05$ & 3.81 \\
-50 & 32 & & 0.023 & $7.37 \mathrm{E}-05$ & 3.80 \\
-38 & -4 & 4768 & 0.063 & $5.24 \mathrm{E}-16$ & 8.02 \\
-54 & 38 & 3768 & 0.051 & $1.90 \mathrm{E}-12$ & 6.94 \\
22 & 44 & 2712 & 0.038 & $1.01 \mathrm{E}-08$ & 5.61 \\
6 & 46 & & 0.027 & $7.55 \mathrm{E}-06$ & 4.33 \\
12 & 44 & & 0.024 & $2.76 \mathrm{E}-05$ & 4.03 \\
-22 & 28 & 1008 & 0.035 & $5.94 \mathrm{E}-08$ & 5.30 \\
\hline
\end{tabular}

Notes: All coordinates are defined in MNI152 space. All statistics listed are significant at $p<0.05$, whole-brain FWE-corrected using a cluster forming threshold of $p<.0001$ uncorrected, and a permutation test with 1000 permutations. 
Table 4 - Significant clusters from the ALE meta-analysis showing a deactivation pattern

\begin{tabular}{|c|c|c|c|c|c|c|c|c|c|}
\hline Cluster \# & Region & Side & $X(\mathrm{~mm})$ & $\mathrm{Y}(\mathrm{mm})$ & $\mathrm{Z}(\mathrm{mm})$ & $\mathrm{mm}^{\wedge} 3$ & ALE & $\mathbf{P}$ & Peak Z \\
\hline \multicolumn{10}{|c|}{ WM deactivation (Control > 2-back) } \\
\hline \multirow[t]{6}{*}{$\# 1$} & posterior cingulate cortex / precuneus & $L / R$ & -4 & -50 & 30 & 5568 & 0.031 & $1.68 \mathrm{E}-11$ & 6.63 \\
\hline & & & -4 & -52 & 12 & & 0.019 & $6.18 \mathrm{E}-07$ & 4.85 \\
\hline & & & 4 & -50 & 18 & & 0.013 & $5.53 \mathrm{E}-05$ & 3.87 \\
\hline & & & -6 & -60 & 16 & & 0.011 & $2.55 \mathrm{E}-04$ & 3.48 \\
\hline & & & 16 & -56 & 30 & & 0.011 & 3.67E-04 & 3.38 \\
\hline & & & 8 & -58 & 20 & & 0.010 & $6.24 \mathrm{E}-04$ & 3.23 \\
\hline \multirow[t]{4}{*}{ \#2 } & ventromedial prefrontal cortex (vmPFC) & $L / R$ & -6 & 58 & 10 & 5480 & 0.028 & $3.27 \mathrm{E}-10$ & 6.18 \\
\hline & & & -6 & 46 & -4 & & 0.020 & $3.88 \mathrm{E}-07$ & 4.94 \\
\hline & & & 4 & 62 & 14 & & 0.016 & 4.97E-06 & 4.42 \\
\hline & & & -2 & 52 & -16 & & 0.010 & $6.57 \mathrm{E}-04$ & 3.21 \\
\hline \#3 & amygdala / hippocampus & $\mathrm{L}$ & -24 & -8 & -22 & 1952 & 0.023 & $1.56 \mathrm{E}-08$ & 5.53 \\
\hline \#4 & amygdala & $\mathrm{R}$ & 24 & -6 & -20 & 1160 & 0.024 & $6.16 \mathrm{E}-09$ & 5.70 \\
\hline \#5 & angular gyrus & $\mathrm{L}$ & -48 & -64 & 28 & 1120 & 0.022 & $3.73 E-08$ & 5.38 \\
\hline \multirow[t]{2}{*}{ \#6 } & middle / superior temporal gyrus & $\mathrm{R}$ & 54 & 4 & -16 & 872 & 0.016 & $1.03 \mathrm{E}-05$ & 4.26 \\
\hline & & & 58 & 4 & -12 & & 0.015 & $1.88 \mathrm{E}-05$ & 4.12 \\
\hline \multicolumn{10}{|c|}{ CR deactivation (Control > Reappraisal) } \\
\hline \multirow[t]{2}{*}{$\# 1$} & amygdala / dorsal entorhinal cortex (BA34) & $\mathrm{R}$ & 26 & -4 & -20 & 3960 & 0.045 & $6.57 \mathrm{E}-15$ & 7.70 \\
\hline & & & 18 & -8 & -16 & & 0.028 & $9.83 \mathrm{E}-09$ & 5.62 \\
\hline$\# 2$ & amygdala / dorsal entorhinal cortex (BA34) & $\mathrm{L}$ & -24 & -6 & -18 & 3000 & 0.058 & $1.78 \mathrm{E}-20$ & 9.20 \\
\hline \#3 & thalamus / parahippocampal gyrus & $\mathrm{L}$ & -22 & -28 & -4 & 688 & 0.026 & 3.77E-08 & 5.38 \\
\hline
\end{tabular}

All coordinates are defined in MNI152 space. All statistics listed are significant at $p<0.05$, whole-brain FWE-corrected using a cluster forming threshold of $p<.0001$ uncorrected, and a permutation test with 1000 permutations. 
Table 5 - Significant clusters from the ALE meta-analysis comparing the deactivation patterns

\begin{tabular}{|c|c|c|c|c|c|c|c|c|c|c|}
\hline Cluster \# & Region & Side & $X(\mathrm{~mm})$ & $Y(\mathrm{~mm})$ & $\mathrm{Z}(\mathrm{mm})$ & $\mathrm{mm}^{\wedge} 3$ & ALE & & $\mathbf{P}$ & Peak Z \\
\hline \multicolumn{11}{|l|}{ Conjunction } \\
\hline$\# 1$ & amygdala & L & -24 & -8 & -22 & 1232 & & 0.023 & na & na \\
\hline \#2 & amygdala & $\mathrm{R}$ & 24 & -6 & -20 & 1064 & & 0.024 & na & na \\
\hline \multicolumn{11}{|c|}{ 2-back > reappraisal } \\
\hline \multirow[t]{4}{*}{$\# 1$} & posterior cingulate cortex / precuneus & $L / R$ & -1 & -51 & 29 & 5048 & & na & $>0.001$ & 3.89 \\
\hline & & & -6 & -49 & 16 & & & na & $1.00 \mathrm{E}-04$ & 3.72 \\
\hline & & & -4 & -56 & 18 & & & na & 8.00E-04 & 3.16 \\
\hline & & & 14 & -56 & 28 & & & na & 0.001 & 3.09 \\
\hline \#2 & ventromedial prefrontal cortex (vmPFC) & $L / R$ & -2 & 59 & 8 & 5008 & & na & $>0.001$ & 3.89 \\
\hline \multirow[t]{2}{*}{ \#3 } & angular gyrus & $\mathrm{L}$ & -49 & -66 & 30 & 1120 & & na & $1.00 \mathrm{E}-04$ & 3.72 \\
\hline & & & -49 & -62 & 23 & & & na & 0.001 & 3.04 \\
\hline \multirow[t]{2}{*}{$\# 4$} & middle temporal gyrus & $\mathrm{R}$ & 53 & 3 & -18 & 872 & & na & $1.00 \mathrm{E}-04$ & 3.72 \\
\hline & & & 58 & 7 & -14 & & & na & 3.00E-04 & 3.43 \\
\hline \#5 & amygdala / hippocampus & $\mathrm{L}$ & -30 & -12 & -24 & 560 & & na & 0.006 & 2.51 \\
\hline \#6 & precuneus & $\mathrm{R}$ & 8 & -58 & 22 & 32 & & na & 0.019 & 2.07 \\
\hline \multicolumn{11}{|c|}{ reappraisal > 2-back } \\
\hline \#1 & amygdala / dorsal entorhinal cortex (BA34) & $\mathrm{L}$ & -24 & 0 & -14 & 616 & & na & 0.004 & 2.64 \\
\hline \#2 & dorsal entorhinal cortex (BA34) & $\mathrm{R}$ & 14 & -6 & -20 & 40 & & na & 0.035 & 1.81 \\
\hline
\end{tabular}

All coordinates are defined in MNI152 space. All statistics listed are significant at $p<0.05$. 


\section{Discussion}

Using a meta-analytic approach, we investigated whether a standard working memory task would downregulate the amygdala similarly to a cognitive reappraisal task. Amygdala deactivation is widely considered as a key neural correlate of cognitive regulation of emotion, and has been documented previously in a meta-analysis of cognitive reappraisal studies (Buhle et al., 2014). We indeed replicate these findings, but critically reveal that a working memory task also robustly triggers deactivation in bilateral clusters in the amygdala, although the extent and topography of the deactivated clusters differed somewhat between the two tasks. Together, our findings suggest that the effects of cognitive reappraisal on the amygdala are driven by cognitive demand rather than the content of the reappraisal.

Downregulation of the amygdala during cognitive reappraisal has typically been interpreted as a top-down inhibition by prefrontal regions (e.g., Etkin et al., 2011). The amygdala is a region critically implicated in threat detection, as has been detailed in animal models (LeDoux, 1996). Indeed, functional MRI studies in humans have revealed activation of the amygdala related to processing of threatening or salient stimuli (Hariri et al., 2002; Morris et al., 1997; Vuilleumier et al., 2001). Via reinterpretation of the threatening situation, with the explicit goal to change the affective impact of the threat, such amygdala reactivity is thought to be reduced. Amygdala downregulation during cognitive reappraisal was furthermore shown to be enhanced by real-time $\mathrm{FMRI}$ neurofeedback based on dIPFC responsivity (Sarkheil et al., 2015). Since there are little or no direct connections between the dIPFC and the amygdala (Amaral et al., 1992), downregulation is thought to occur indirectly via the ventromedial prefrontal cortex (Buhle et al., 2014), a region involved in implicit forms of emotion regulation such as extinction learning (Hartley and Phelps, 2010). Thus, the commonly held view is that the act of cognitive reappraisal, through neural pathways that are shared with other emotion regulation strategies, leads to a downregulation of the amygdala reactivity to threat.

However, our findings demonstrate that a standard working memory task is also accompanied by a downregulation of the amygdala. This suggests that the content of the cognitive task may not be relevant. While at odds with theories of cognitive reappraisal, this notion is in line theories postulating a reciprocal relationship between large-scale neural systems encompassing dIPFC (the executive control network) and amygdala (the salience network; Hermans et al., 2014). For instance, acute threat is known to trigger activation of 
the salience network, and this is accompanied by a loss of executive control network function (Hermans et al., 2014). Most evidence for this comes from studies that have investigated the impact of acute threat and arousal on executive functioning. For example, behavioral studies have shown that during high states of arousal, working memory performance is impaired (Elzinga and Roelofs, 2005; Lupien et al., 1999). This trade-off also occurs at the network level, namely, when participants perform a working memory task while under threat, BOLD signal in the executive control network is reduced compared to a non-threatening context (Van Ast et al., 2016). Furthermore, the dynamics between the salience network and the central executive control network was shown to change during acute threat (Young et al., 2017).

Our findings suggest that such a trade-off between the salience network and the executive control network may also occur the other way around. This idea is in line with previous studies indicating that defensive responses which have shown to be (partly) dependent on the amygdala (Bechara et al., 1995; Klumpers et al., 2015; LaBar et al., 1995), are reduced during cognitively demanding tasks. For instance, during working memory maintenance, threat conditioning is impaired (Carter et al., 2003), and threat-potentiated startle responses are decreased (Vytal et al., 2012). Other types of cognitively demanding tasks, apart from the 2-back working memory task we investigated here, also downregulate the amygdala. Examples are playing a game of Tetris (Price et al., 2013) or making goaldirected eye movements (de Voogd et al., 2018b; Jamadar et al., 2013). Cognitive demand may indeed lead to a competition between the executive control network and the salience network, where resources are allocated to the executive control network at the expense of the salience network (de Voogd et al., 2018a). Thus, the reduced BOLD signal found in the amygdala during cognitive reappraisal and working memory tasks is in line with a vast body of literature showing reciprocal relationships between large-scale neural systems.

If the executive control network and the salience network are reciprocally activated with respect to one another in both directions, an important question that remains to be answered is how this competitive allocation of resources is established. A first possibility is that resource allocation is established via active suppression. This may occur during a working memory task in a similar fashion as has been proposed for cognitive reappraisal. Namely, downregulation of the amygdala may occur indirectly via the vmPFC (Buhle et al., 2014). This mechanism is similar to the proposed working mechanism of implicit emotion regulation such as extinction learning (Hartley and Phelps, 2010), since during extinction, it has been shown 
the amygdala is inhibited by the ventromedial prefrontal cortex (vmPFC), leading to a reduction in the expression of threat responses (Milad and Quirk, 2012). Indeed, it has been proposed that the vmPFC may serve as a common mechanism for reducing learned defensive responses to threat (Schiller and Delgado, 2010). This pathway may be activated via several pathways including those involved in high-order cognition such as the dIPFC, and our findings suggest that the specific content of the cognitive process may not be a critical factor.

It is worthwhile to also consider other potential explanations for the reciprocal relationship between dIPFC and amygdala as observed using functional MRI. One alternative possibility is that when one large-scale network activates, an increase in blood flow to those regions may deplete other neural systems from of oxygenated blood, resulting in decreased BOLD-fMRI signal. Recent findings indicate that BOLD signal in specific functional brain networks may indeed be partly driven by vascular regulation (Bright et al., 2020). The fact that alterations in amygdala-dependent functions are seen during cognitively demanding tasks that elicit reduced BOLD in the amygdala (Carter et al., 2003; de Voogd et al., 2018a, 2018b; Fox et al., 2009; Hermans et al., 2014) appears to speak against the notion that this BOLD signal decrease is a purely vascular effect. However, it is also possible that depletion of oxygenated blood may itself affect neuronal activity. There is indeed evidence that vascular changes can influence neuronal activity (Croal et al., 2015; Hall et al., 2011). Future studies should therefore determine whether amygdala downregulation during cognitively demanding tasks is also observed using electrophysiological methods, which more directly measure neuronal activity.

If a cognitively demanding task can reduce threat-related processes (Carter et al., 2003; Vytal et al., 2012) via downregulation of the amygdala, this may have clinical implications. Interestingly, a cognitively demanding task that involves goal-directed attention may already be part of a therapy, namely, in the case of Eye Movement Desensitization and Reprocessing (EMDR; Shapiro, 1989). EMDR is an evidence-based therapy for treatment of fear and anxiety-related disorders (Bisson et al., 2013; Lee and Cuijpers, 2013). This therapy has been widely used in clinical populations, but a mechanistic understanding of the role of eye movements in this therapy is still largely unclear. Laboratory studies have shown that making cognitively demanding eye movements (de Voogd et al., 2018b) or a working memory task (de Voogd and Phelps, 2020; Loos et al., 2020) embedded during extinction learning reduces defensive responses to threat in healthy (de Voogd et al., 2018b; de Voogd and 
Phelps, 2020) and phobic (Loos et al., 2020) participants. These cognitively demanding tasks during extinction learning were accompanied by downregulation of the amygdala (de Voogd et al., 2018b; Loos et al., 2020). It could therefore be the case that an additional inhibition of the amygdala during extinction can strengthen safety learning.

If indeed cognitive demand is the mechanism underlying cognitive reappraisal, then any task that is cognitively demanding may potentially be a suitable intervention to reduce defensive responses to threat, and potentially have added value in a clinical setting. An ideal intervention, however, should allow for the cognitive demand to be systematically increased to accommodate individual differences in cognitive capacity. The cognitive demand of a working memory task can be systematically increased and has a greater impact on the reduction of BOLD signal in the amygdala (de Voogd et al., 2018a). In comparison to cognitive reappraisal, which is one of the most common cognitive emotion regulation strategies translated to the clinic (Kredlow et al., 2020), compliance with task instructions and task performance in working memory tasks are easier to assess. Moreover, working memory tasks typically impair episodic memory for threatening events (Onderdonk and van den Hout, 2016), while cognitive reappraisal typically enhances episodic memory for threatening events (Dillon et al., 2007; Hayes et al., 2010), likely due to increased attention and encoding (Hayes et al., 2010). Since our findings indicate that they operate via similar neural pathways, working memory tasks may have benefits over cognitive reappraisal as a treatment intervention.

It has been argued that distraction during exposure may be counterproductive as it leads to avoidance. It may therefore be the case that performing a cognitively demanding task during treatment may induce distraction and thereby avoidance. However, empirical evidence suggest that in some cases, distraction may be more beneficial than focused exposure (see, for a review, Podină et al., 2013). Moreover, goal-directed eye movements as used in EMDR could also be seen as distraction, but have been shown to have beneficial effects on threat-related symptoms compared to exposure or extinction alone (de Voogd et al., 2018b; de Voogd and Phelps, 2020; Lee and Cuijpers, 2013).

We observed that only a subset of the articles included in our meta-analysis reported a deactivation contrast. This was the case for the 2-back working memory studies (i.e., 16 out of 66 studies) and the cognitive reappraisal studies (i.e., 29 out of 65 studies). It is possible that underreporting of deactivation contrasts has consequences for the conclusion of our findings. We cannot rule out that a systematic bias has led to the decision to report or not to 
report deactivation patterns. It may be that studies that have reported deactivation patterns may have done so because the results were in line with the expectation. This may be specifically true for cognitive reappraisal studies, as amygdala downregulation forms an important part of the mechanistic explanation of how reappraisal is established. Moreover, we observed that from the studies that contributed to the amygdala deactivation during cognitive reappraisal, 12 out of the 16 reported amygdala deactivations based on Small Volume Correction (SVC), while only one of the six studies that contributed to the amygdala deactivation during working memory reported amygdala deactivation based on SVC. It is therefore possible that this bias has led to an overrepresentation of amygdala deactivation for cognitive reappraisal. We propose that patterns of downregulation are meaningful and that it is therefore important to report BOLD deactivation patterns as well. This will ultimately contribute to a broader understanding of the role of network dynamics in the brain and its relation to function.

Although we observed a striking overlap in amygdala deactivation between working memory and cognitive reappraisal, we also observed that the overlap was not absolute. We observed two deactivation clusters in the left amygdala that were unique for either cognitive reappraisal or working memory. For cognitive reappraisal, this deactivation was located dorsally with respect to the conjunction deactivation, within the amygdala and Brodmann area 34. For working memory, the location of the deactivation was more ventral, within the amygdala and hippocampus. This can be interpreted in a few ways. First, it is possible that the deactivation across the two tasks is not identical and both lead to a deactivation pattern that is unique to the task that is being conducted. Second, an alternative explanation could be that the difference is due to a bias in reporting. Since the amygdala deactivation during cognitive reappraisal is largely based on an SVC, it is possible that this influences the location of the reported peak voxel (i.e., this would always lie within the amygdala). Several studies have shown that deactivation patterns during a working memory task are present in both amygdala and hippocampus (Cousijn et al., 2010; de Voogd et al., 2018b; Qin et al., 2009). It is therefore possible that with an SVC, the reporting of the peak value is more biased towards the hippocampus in working memory studies. To resolve this, a study directly comparing working memory and cognitive reappraisal would be necessary to investigate whether the deactivation patterns are similar or meaningfully distinct. 
In conclusion, using meta-analytic evidence, we demonstrate that both cognitive reappraisal tasks and working memory tasks deactivate the amygdala, thus suggesting that the amygdala deactivation is driven by cognitive demand rather than the actual reinterpretation of a threatening stimulus. Our findings are in line with accounts of brain function in terms of reciprocal activation or competition between large-scale neural networks.

\section{Acknowledgements}

We thank Lieke Martens, Habiba Schiller, and Zhiwei Zheng for their assistance. This work was supported by the European Research Council (ERC-2015- CoG 682591) awarded to EJH.

\section{Conflict of interest}

The authors report no conflict of interest. 


\section{References}

Amaral, D.G., Price, J.L., Pitkanen, A., Carmichael, S.T., 1992. Anatomical organization of the primate amygdaloid complex., in: The Amygdala: Neurobiological Aspects of Emotion, Memory, and Mental Dysfunction.

Balderston, N.L., Quispe-Escudero, D., Hale, E., Davis, A., O’Connell, K., Ernst, M., Grillon, C., 2016. Working memory maintenance is sufficient to reduce state anxiety. Psychophysiology. https://doi.org/10.1111/psyp.12726

Bechara, A., Tranel, D., Damasio, H., Adolphs, R., Rockland, C., Damasio, A.R., 1995. Double dissociation of conditioning and declarative knowledge relative to the amygdala and hippocampus in humans. Science (80-. ). https://doi.org/10.1126/science.7652558

Bisson, J.I., Roberts, N.P., Andrew, M., Cooper, R., Lewis, C., 2013. Psychological therapies for chronic post-traumatic stress disorder ( PTSD ) in adults. Cochrane Database Syst. Rev. 4-7.

Bright, M.G., Whittaker, J.R., Driver, I.D., Murphy, K., 2020. Vascular physiology drives functional brain networks. Neuroimage. https://doi.org/10.1016/j.neuroimage.2020.116907

Buhle, J.T., Silvers, J.A., Wage, T.D., Lopez, R., Onyemekwu, C., Kober, H., Webe, J., Ochsner, K.N., 2014. Cognitive reappraisal of emotion: A meta-analysis of human neuroimaging studies. Cereb. Cortex. https://doi.org/10.1093/cercor/bht154

Carter, R.M., Hofstotter, C., Tsuchiya, N., Koch, C., 2003. Working memory and fear conditioning. Proc. Natl. Acad. Sci. 100, 1399-1404.

https://doi.org/10.1073/pnas.0334049100

Cousijn, H., Rijpkema, M., Qin, S., van Marle, H.J.F., Franke, B., Hermans, E.J., van Wingen, G., Fernandez, G., 2010. Acute stress modulates genotype effects on amygdala processing in humans. Proc. Natl. Acad. Sci. U. S. A. 107, 9867-9872.

https://doi.org/10.1073/pnas.1003514107

Croal, P.L., Hall, E.L., Driver, I.D., Brookes, M.J., Gowland, P.A., Francis, S.T., 2015. The effect of isocapnic hyperoxia on neurophysiology as measured with MRI and MEG.

Neuroimage. https://doi.org/10.1016/j.neuroimage.2014.10.036

de Voogd, L.D., Hermans, E.J., Phelps, E.A., 2018a. Regulating defensive survival circuits through cognitive demand via large-scale network reorganization. Curr. Opin. Behav. Sci. 24, 124-129. https://doi.org/10.1016/j.cobeha.2018.08.009

de Voogd, L.D., Kanen, J.W., Neville, D.A., Roelofs, K., Fernández, G., Hermans, E.J., 2018b. Eye-movement intervention enhances extinction via amygdala deactivation. J. Neurosci. 38, 8694-8706. https://doi.org/10.1523/JNEUROSCI.0703-18.2018

de Voogd, L.D., Phelps, E.A., 2020. A cognitively demanding working-memory intervention enhances extinction 1-11. https://doi.org/10.1038/s41598-020-63811-0

Dillon, D.G., Ritchey, M., Johnson, B.D., LaBar, K.S., 2007. Dissociable effects of conscious emotion regulation strategies on explicit and implicit memory. Emotion 7, 354-365. https://doi.org/10.1037/1528-3542.7.2.354

Eickhoff, S.B., Bzdok, D., Laird, A.R., Kurth, F., Fox, P.T., 2012. Activation likelihood estimation meta-analysis revisited. Neuroimage. https://doi.org/10.1016/j.neuroimage.2011.09.017

Eickhoff, S.B., Laird, A.R., Grefkes, C., Wang, L.E., Zilles, K., Fox, P.T., 2009. Coordinate-based activation likelihood estimation meta-analysis of neuroimaging data: A random-effects approach based on empirical estimates of spatial uncertainty. Hum. Brain Mapp. 
https://doi.org/10.1002/hbm.20718

Elzinga, B.M., Roelofs, K., 2005. Cortisol-induced impairments of working memory require acute sympathetic activation. Behav. Neurosci. https://doi.org/10.1037/07357044.119.1.98

Etkin, A., Egner, T., Kalisch, R., 2011. Emotional processing in anterior cingulate and medial prefrontal cortex. Trends Cogn. Sci. https://doi.org/10.1016/j.tics.2010.11.004

Fox, M.D., Zhang, D., Snyder, A.Z., Raichle, M.E., 2009. The global signal and observed anticorrelated resting state brain networks. J. Neurophysiol. https://doi.org/10.1152/jn.90777.2008

Hall, E.L., Driver, I.D., Croal, P.L., Francis, S.T., Gowland, P.A., Morris, P.G., Brookes, M.J., 2011. The effect of hypercapnia on resting and stimulus induced MEG signals. Neuroimage. https://doi.org/10.1016/j.neuroimage.2011.06.073

Hariri, A.R., Tessitore, A., Mattay, V.S., Fera, F., Weinberger, D.R., 2002. The amygdala response to emotional stimuli: a comparison of faces and scenes. Neuroimage 17, 317323. https://doi.org/10.1006/nimg.2002.1179

Hartley, C.A., Phelps, E.A., 2010. Changing fear: The neurocircuitry of emotion regulation. Neuropsychopharmacology. https://doi.org/10.1038/npp.2009.121

Hayes, J.P., Morey, R.A., Petty, C.M., Seth, S., Smoski, M.J., McCarthy, G., LaBar, K.S., 2010. Staying Cool when Things Get Hot: Emotion Regulation Modulates Neural Mechanisms of Memory Encoding. Front. Hum. Neurosci. https://doi.org/10.3389/fnhum.2010.00230

Hermans, E.J., Henckens, M.J.A.G., Joëls, M., Fernández, G., 2014. Dynamic adaptation of large-scale brain networks in response to acute stressors. Trends Neurosci. 37, 304314. https://doi.org/10.1016/j.tins.2014.03.006

Jamadar, S.D., Fielding, J., Egan, G.F., 2013. Quantitative meta-analysis of fMRI and PET studies reveals consistent activation in fronto-striatal-parietal regions and cerebellum during antisaccades and prosaccades. Front. Psychol. 4, 1-15. https://doi.org/10.3389/fpsyg.2013.00749

King, R., Schaefer, A., 2011. The emotional startle effect is disrupted by a concurrent working memory task. Psychophysiology. https://doi.org/10.1111/j.14698986.2010.01062.x

Klumpers, F., Morgan, B., Terburg, D., Stein, D.J., van Honk, J., 2015. Impaired acquisition of classically conditioned fear-potentiated startle reflexes in humans with focal bilateral basolateral amygdala damage. Soc. Cogn. Affect. Neurosci. 5, 1611-1168. https://doi.org/10.1093/scan/nsu164

Kohn, N., Eickhoff, S.B., Scheller, M., Laird, A.R., Fox, P.T., Habel, U., 2014. Neural network of cognitive emotion regulation - An ALE meta-analysis and MACM analysis. Neuroimage. https://doi.org/10.1016/j.neuroimage.2013.11.001

Kredlow, M.A., de Voogd, L.D., Phelps, E.A., 2020. Laboratory Analogues and Therapy Procedures: A Case for Translation from the Clinic to the Laboratory. PsyArXiv.

LaBar, K.S., LeDoux, J.E., Spencer, D.D., Phelps, E.A., 1995. Impaired fear conditioning following unilateral temporal lobectomy in humans. J. Neurosci.

https://doi.org/10.1523/jneurosci.15-10-06846.1995

LeDoux, J.E., 1996. The Emotional Brain. Simon and Schuster, New York.

Lee, C.W., Cuijpers, P., 2013. A meta-analysis of the contribution of eye movements in processing emotional memories. J. Behav. Ther. Exp. Psychiatry 44, 231-239. https://doi.org/10.1016/j.jbtep.2012.11.001 
Lee, T.W., Xue, S.W., 2018. Does emotion regulation engage the same neural circuit as working memory? A meta-analytical comparison between cognitive reappraisal of negative emotion and 2-back working memory task. PLoS One.

https://doi.org/10.1371/journal.pone.0203753

Loos, E., Schicktanz, N., Fastenrath, M., Coynel, D., Milnik, A., Fehlmann, B., Egli, T., Ehrler, M., Papassotiropoulos, A., de Quervain, D.J.-F., 2020. Reducing Amygdala Activity and Phobic Fear through Cognitive Top-Down Regulation. J. Cogn. Neurosci. 1-13. https://doi.org/10.1162/jocn_a_01537

Lupien, S.J., Gillin, C.J., Hauger, R.L., 1999. Working memory is more sensitive than declarative memory to the acute effects of corticosteroids: A dose-response study in humans. Behav. Neurosci. https://doi.org/10.1037/0735-7044.113.3.420

McRae, K., Hughes, B., Chopra, S., Gabrieli, J.D.E., Gross, J.J., Ochsner, K.N., 2010. The Neural Bases of Distraction and Reappraisal. J. Cogn. Neurosci. 22, 248-262. https://doi.org/10.1162/jocn.2009.21243

Milad, M.R., Quirk, G.J., 2012. Fear Extinction as a Model for Translational Neuroscience: Ten Years of Progress. Annu. Rev. Psychol. 63, 129-151. https://doi.org/10.1146/annurev.psych.121208.131631

Moher, D., Shamseer, L., Clarke, M., Ghersi, D., Liberati, A., Petticrew, M., Shekelle, P., Stewart, L.A., Estarli, M., Barrera, E.S.A., Martínez-Rodríguez, R., Baladia, E., Agüero, S.D., Camacho, S., Buhring, K., Herrero-López, A., Gil-González, D.M., Altman, D.G., Booth, A., Chan, A.W., Chang, S., Clifford, T., Dickersin, K., Egger, M., Gøtzsche, P.C., Grimshaw, J.M., Groves, T., Helfand, M., Higgins, J., Lasserson, T., Lau, J., Lohr, K., McGowan, J., Mulrow, C., Norton, M., Page, M., Sampson, M., Schünemann, H., Simera, I., Summerskill, W., Tetzlaff, J., Trikalinos, T.A., Tovey, D., Turner, L., Whitlock, E., 2016. Preferred reporting items for systematic review and meta-analysis protocols (PRISMAP) 2015 statement. Rev. Esp. Nutr. Humana y Diet. https://doi.org/10.1186/2046-40534-1

Morris, J.S., Friston, K.J., Dolan, R.J., 1997. Neural responses to salient visual stimuli. Proc. Biol. Sci. 264, 769-775. https://doi.org/10.1098/rspb.1997.0109

Ochsner, K.N., Silvers, J.A., Buhle, J.T., 2012. Functional imaging studies of emotion regulation: a synthetic review and evolving model of the cognitive control of emotion. Ann. N. Y. Acad. Sci. https://doi.org/10.1111/j.1749-6632.2012.06751.x

Öhman, A., 2005. The role of the amygdala in human fear: Automatic detection of threat. Psychoneuroendocrinology. https://doi.org/10.1016/j.psyneuen.2005.03.019

Onderdonk, S.W., van den Hout, M.A., 2016. Comparisons of eye movements and matched changing visual input. J. Behav. Ther. Exp. Psychiatry 53, 34-40.

https://doi.org/10.1016/j.jbtep.2015.10.010

Podină, I.R., Koster, E.H.W., Philippot, P., Dethier, V., David, D.O., 2013. Optimal attentional focus during exposure in specific phobia: A meta-analysis. Clin. Psychol. Rev. https://doi.org/10.1016/j.cpr.2013.10.002

Price, R.B., Paul, B., Schneider, W., Siegle, G.J., 2013. Neural correlates of three neurocognitive intervention strategies: A preliminary step towards personalized treatment for psychological disorders. Cognit. Ther. Res. 37, 657-672. https://doi.org/10.1007/s10608-012-9508-x

Qin, S., Hermans, E.J., van Marle, H.J.F., Luo, J., Fernández, G., 2009. Acute psychological stress reduces working memory-related activity in the dorsolateral prefrontal cortex. Biol. Psychiatry 66, 25-32. https://doi.org/10.1016/j.biopsych.2009.03.006 
Sarkheil, P., Zilverstand, A., Kilian-Hütten, N., Schneider, F., Goebel, R., Mathiak, K., 2015. fMRI feedback enhances emotion regulation as evidenced by a reduced amygdala response. Behav. Brain Res. https://doi.org/10.1016/j.bbr.2014.11.027

Schiller, D., Delgado, M.R., 2010. Overlapping neural systems mediating extinction, reversal and regulation of fear. Trends Cogn. Sci. https://doi.org/10.1016/j.tics.2010.04.002

Seeley, W.W., Menon, V., Schatzberg, A.F., Keller, J., Glover, G.H., Kenna, H., Reiss, A.L., Greicius, M.D., 2007. Dissociable Intrinsic Connectivity Networks for Salience Processing and Executive Control. J. Neurosci. 27, 2349-2356. https://doi.org/10.1523/JNEUROSCI.5587-06.2007

Shapiro, F., 1989. Eye movement desensitization: A new treatment for post-traumatic stress disorder. J. Behav. Ther. Exp. Psychiatry 20, 211-217. https://doi.org/10.1016/00057916(89)90025-6

Turkeltaub, P.E., Eickhoff, S.B., Laird, A.R., Fox, M., Wiener, M., Fox, P., 2012. Minimizing within-experiment and within-group effects in activation likelihood estimation metaanalyses. Hum. Brain Mapp. https://doi.org/10.1002/hbm.21186

Van Ast, V.A., Spicer, J., Smith, E.E., Schmer-Galunder, S., Liberzon, I., Abelson, J.L., Wager, T.D., 2016. Brain Mechanisms of Social Threat Effects on Working Memory. Cereb. Cortex. https://doi.org/10.1093/cercor/bhu206

Vuilleumier, P., Armony, J.L., Driver, J., Dolan, R.J., 2001. Effects of attention and emotion on face processing in the human brain: an event-related fMRI study. Neuron 30, 829-841. https://doi.org/S0896-6273(01)00328-2 [pii]

Vytal, K., Cornwell, B., Arkin, N., Grillon, C., 2012. Describing the interplay between anxiety and cognition: from impaired performance under low cognitive load to reduced anxiety under high load. Psychophysiology 49, 842-52. https://doi.org/10.1111/j.14698986.2012.01358.x

Wager, T.D., Smith, E.E., 2003. Neuroimaging studies of working memory: A meta-analysis, Cognitive, Affective and Behavioral Neuroscience. https://doi.org/10.3758/CABN.3.4.255

Young, C.B., Raz, G., Everaerd, D., Beckmann, C.F., Tendolkar, I., Hendler, T., Fernández, G., Hermans, E.J., 2017. Dynamic Shifts in Large-Scale Brain Network Balance As a Function of Arousal. J. Neurosci. https://doi.org/10.1523/JNEUROSCI.1759-16.2016 